\title{
Sulfarotene, a synthetic retinoid, overcomes stemness and sorafenib resistance of hepatocellular carcinoma via suppressing SOS2-RAS pathway
}

Feng Qi ${ }^{1,2+}$, Wenxing Qin ${ }^{3 \dagger}$, Yao Zhang ${ }^{4 \dagger}$, Yongde Luo ${ }^{5,6}$, Bing Niư ${ }^{7}$, Quanlin An ${ }^{1}$, Biwei Yang ${ }^{1,2}$, Keqing Shi ${ }^{5}$, Zhijie $\mathrm{Yu}^{5}$, Junwei Chen ${ }^{4^{*}}$ (D, Xin Cao ${ }^{1 *}$ (D) and Jinglin Xia ${ }^{1,2,5^{*}}$ (iD)

\begin{abstract}
Background: Recurrent hepatocellular carcinoma (HCC) shows strong resistance to sorafenib, and the tumorrepopulating cells (TRCs) with cancer stem cell-like properties are considered a driver for its high recurrent rate and drug resistance.

Methods: Suppression of TRCs may thus be an effective therapeutic strategy for treating this fatal disease. We evaluated the pharmacology and mechanism of sulfarotene, a new type of synthetic retinoid, on the cancer stem cell-like properties of HCC TRCs, and assessed its preclinical efficacy in models of HCC patient-derived xenografts (PDXs).

Results: Sulfarotene selectively inhibited the growth of HCC TRCs in vitro and significantly deterred TRC-mediated tumor formation and lung metastasis in vivo without apparent toxicity, with an $I_{50}$ superior to that of acyclic retinoid and sorafenib, to which the recurrent HCC exhibits significant resistance at advanced stage. Sulfarotene promoted the expression and activation of RARa, which down-regulated SOS2, a key signal mediator associated with RAS activation and signal transduction involved in multiple downstream pathways. Moreover, sulfarotene selectively inhibited tumorigenesis of HCC PDXs with high expression for SOS2.

Conclusions: Our study identified sulfarotene as a selective inhibitor for the TRCS of HCC, which targets a novel RARa-SOS2-RAS signal nexus, shedding light on a new, promising strategy of target therapy for advanced liver cancer.
\end{abstract}

Keywords: Hepatocellular carcinoma, Tumor-repopulating cells, Retinoid, Sulfarotene, SOS2

\footnotetext{
* Correspondence: chenjunwei@hust.edu.cn; caox@fudan.edu.cn;

xiajinglin@fudan.edu.cn

${ }^{\dagger}$ Feng Qi, Wenxing Qin and Yao Zhang contributed equally to this work.

${ }^{4}$ Laboratory for Cellular Biomechanics and Regenerative Medicine,

Department of Biomedical Engineering, College of Life Science and

Technology, Huazhong University of Science and Technology, 1037 Luoyu

Road, 430074 Wuhan, Hubei, China

${ }^{1}$ Institute of Clinical Science, Zhongshan Hospital, Fudan University, 180

Fenglin Road, 200032 Shanghai, China

Full list of author information is available at the end of the article
}

(c) The Author(s). 2021 Open Access This article is licensed under a Creative Commons Attribution 4.0 International License, which permits use, sharing, adaptation, distribution and reproduction in any medium or format, as long as you give appropriate credit to the original author(s) and the source, provide a link to the Creative Commons licence, and indicate if changes were made. The images or other third party material in this article are included in the article's Creative Commons licence, unless indicated otherwise in a credit line to the material. If material is not included in the article's Creative Commons licence and your intended use is not permitted by statutory regulation or exceeds the permitted use, you will need to obtain permission directly from the copyright holder. To view a copy of this licence, visit http://creativecommons.org/licenses/by/4.0/ The Creative Commons Public Domain Dedication waiver (http://creativecommons.org/publicdomain/zero/1.0/) applies to the data made available in this article, unless otherwise stated in a credit line to the data. 


\section{Background}

Hepatocellular carcinoma (HCC) is the main type of liver cancer and ranks fifth among the most common malignancies worldwide. The incidence of $\mathrm{HCC}$ is expected to continue rising to become the second leading cause of cancer-related deaths [1]. Although several treatment strategies for HCC are currently used in clinical practice [2], the high recurrence rate and emerging drug resistance contribute significantly to this dire situation [3].

Cancer stem cells (CSCs) or tumor-initiating cells (TICs) are a small, specialized subpopulation of cells that arise from the precancerous lesion, which play a predominant role in the progression, recurrence and resistance to therapy of HCC [4]. Instead of the complex approaches used for the identification and isolation of CSCs or TICs, e.g. by sets of stem cell surface markers [5], that hamper study applicability, we adapted and developed a simple mechanical approach to select cancer stem-like cells while they were cultured in threedimensional (3D) soft fibrin gels [6] rather than twodimensional (2D) rigid dishes. Such selectively survived cancer stem-like cells were shown to be highly tumorigenic, and thus were defined as tumor-repopulating cells (TRCs) [6, 7]. In a previous study, our group identified a new synthetic retinoid, named sulfarotene (WYC-209), that exerted potent, selective activity in suppressing the growth and tumor-initiating ability of TRCs derived from various types of cancer with negligible toxicity [8]. The results suggested that the combination of sulfarotene with the efficient TRC selection for cancer stemlike cells could be used to better tackle the current HCC problems as aforementioned.

In the present study, we have found that sulfarotene selectively inhibited TRCs of HCC origins and metastatic tumor formation in multiple preclinical models, including TRCs-based xenografts and patient derived xenografts (PDXs). Of note, sulfarotene effectively suppressed tumor formation and lung metastasis of the HCC TRCs that otherwise were resistance to sorafenib and ACR. Mechanistically, we demonstrated that sulfarotene upregulated RAR $\alpha$ in HCC TRCs, which downregulated SOS2, an important mediator of oncogenic RAS activation that is critical for multiple upstream as well as downstream signaling pathways. Thus, our study has identified sulfarotene as a potential therapeutic agent for treating the TRCs of HCC by targeting a novel RAR $\alpha$ SOS2-RAS signal axis, which plays critical roles in tumorigenicity of TRCs and mediation of the therapeutic effects of sulfarotene.

\section{Materials and methods}

\section{TRCs proliferation, migration and invasion assay}

TRCs cultured in 3D fibrin gels were treated with agents, after $24 \mathrm{~h}$, cell viability was assessed by using the
Cell Counting Kit-8 (CCK-8, Dojindo, Japan) according to the manufacturer's protocol in which the absorbance of produced formazan dye was measured at $450 \mathrm{~nm}$. The cell inhibition rate was calculated according to the formula [(OD control cells - OD treated cells) / (OD control cells - OD blanks)] $\times 100$. The capacity of cell migration was determined by transwell migration and invasion assay as previously described [9].

\section{PDX, orthotopic transplant mouse models and lung metastasis mouse models}

Tumor tissues freshly isolated from patients in the operating room were dissected into small blocks of 1 $\mathrm{mm}^{3}$ under aseptic conditions. In a sterile environment, NOG mice were anesthetized and the HCC tissue blocks were implanted subcutaneously into the top right flank of the mice. About two months later, after reaching $1 \mathrm{~cm}$ in diameter, the subcutaneous PDX tumor nodes were removed, dissected into approximately $2 \times 2 \times 2 \mathrm{~mm}^{3}$ pieces, and re-transplanted into the flanks of nude mice for 30 days to permit growth as previously described $[10,11]$.

The mice were euthanized at day 30 or when a tumor node reached $15 \mathrm{~mm}$ in diameter. In metastasis experiments, the TRCs and treatment agents were injected i.v. through the tail vein into BALB/C nude mice. The lung was dissected and subjected to bioluminescence imaging in IVIS Lumina II with image radiance values normalized by the Living Image program (PerkinElmer, Boston, MA). The weight of the metastatic lung was measured and the metastasis rate was determined on paraffin embedded and HE stained lung sections by counting detectable tumor foci. PLC/PRF/5-TRCs stably overexpressing SOS2 or shRNA targeting SOS2 (see details in the Supplemental Materials) were established by puromycin antibiotic selection. The inoculation of these SOS2manipulated TRCs into nude mice and subsequent drug treatment schemes essentially followed the methods described above.

\section{Identification of critical responsive genes by ranking and network analyses}

Critical genes responsive to sulfarotene treatment were selected and ranked based on an integrated analysis of RNA-Seq and ChIP-Seq data according to the priority criteria as follows: (1) degree of neighbor nodes. The correlation of the integrated genes obtained from RNA-Seq and ChIP-Seq data ( $\mid$ PCCs $\mid>$ 0.9 and $P<0.05)$ was determined by Pearson's correlation coefficients (PCCs) analysis, from which a molecular network was constructed with differential degrees determined by the number of neighboring genes linked to each critical gene that was 
considered to play an important role in the network; (2) total number of involved pathways. After selection of a top list of associated genes according to the degree value $(\geq 18)$, critical genes were differentially ranked based on the number of the involved biological pathways, especially those closely related to tumorigenesis, e.g., the RAS signaling pathway and pathways associated with cell cycle progression, pluripotency of stem cells and ubiquitin mediated proteolysis in the KEGG annotated pathways [12]; (3) ChIP-Seq peak values, which indicate expression values of genes in association with RAR $\alpha$ with statistic significance (M-value $\geq 0.5, P$-value $<0.05)$; (4) the membership in 3 clusters. Assuming genes downregulated in response to sulfarotene treatment as having priority roles as oncogenes, genes enriched in clusters 1,3 and 5 based on the change patterns were considered as targets for sulfarotene treatment with a membership score $\geq 0.2$.

\section{HCC patients recruitment and follow-up}

A total of 282 patients with HCC who underwent curative resection between January 2009 and January 2010 were enrolled in 2 independent cohorts at the First Affiliated Hospital of Wenzhou Medical University (Zhejiang, China). All patients were identified by the pathologic diagnosis of HCC who had not yet received drug treatment. The clinicopathological data of all patients included the grade, stage and tumor location. However, among all the patients, 45 in cohort 1 had no follow-up while 237 were monitored until May 1, 2019, of whom 127 experienced postoperative pulmonary metastasis, as previously described [11]. Each patient provided informed consent before they participated in the study. The study protocol was approved by the Ethics Committee of The First Affiliated Hospital of Wenzhou Medical University (Zhejiang, China). The overall survival (OS) was defined as the duration from surgery to death or final follow-up, while the time to recurrence (TTR) was defined as the interval between the surgery and recurrence.

\section{Results}

Sulfarotene selectively targets tumor-repopulating cells of $\mathrm{HCC}$ in vitro and in vivo

First, sulfarotene was used to screen HCC cell lines, and effectively inhibited the proliferation of Hep3B and PLC/PRF/5 cells among the less invasive cells (Figure S1). Then, we established the TRC model with two human HCC cell lines, Hep3B and PLC/ $\mathrm{PRF} / 5$, as previously described [6]. In view of these two HCC cell lines are weaker than other HCC cell lines in terms of proliferation and invasion, the selected TRCs from Hep3B and PLC/PRF/5 cells were more stem-like compared with their parental cancer cells. FACS analyses revealed that 89.2 and $82.1 \%$ of the resulting Hep3B-TRCs and PLC/PRF/ 5-TRCs, respectively, were enriched for the expression of $\mathrm{EpCAM}^{+}$and $\mathrm{CD} 133^{+}$, two surface antigens that mark the stemness of HCC stem cells [13] (Figure S2a, b). Transwell assays revealed significantly increased survival and migration of these TRCs over their parental cancer cells in vitro (Figure S2c). Furthermore, all inoculums of both types of TRCs, sampled at $5 \times 10^{5}$ cells selected from initial parental cancer cells, formed significant subcutaneous xenograft tumor nodes in BALB/c nude mice compared to none of their parental cancer cells at 30 days (Figure S2d, e), confirming the notable selfrenewal and tumorigenic properties of the isolated TRCs.

Using these HCC TRC models, we evaluated the therapeutic effects of the recently discovered RA analog sulfarotene (Figure S3) [8] in comparison with ACR, the open-ring RA analog developed almost three decades ago, sorafenib, and the solvent carrier DMSO as the negative control. After treatment for 5 days, we found that the colony spheroids from HCC TRCs were markedly suppressed by sulfarotene at concentrations ranging from 1.0 to 10 $\mu \mathrm{M}$, compared to $10 \mu \mathrm{M}$ for ACR or sorafenib (Fig. 1a). Similarly, sulfarotene caused a significantly higher percent of apoptotic cell deaths in both types of HCC-related TRCs compared to the other two drugs tested (Fig. 1b and Figure S4a).

Sulfarotene exhibited the lowest $\mathrm{IC}_{50}$ values of 2.83 and $1.08 \mu \mathrm{M}$ of the selected Hep3B-TRCs and PLC/ PRF/5-TRCs after $48 \mathrm{~h}$ treatment, which increased to 10.20 and $6.04 \mu \mathrm{M}$ for Hep3B and PLC/PRF/5 cancer cells (Fig. $1 \mathrm{c}$ and Figure S4b). However, the $\mathrm{IC}_{50}$ values of sorafenib increased 2.5 and 10 fold for these two types of TRCs, respectively, which is in accordance with the reported sorafenib resistance of CSCs and in the clinic [14]. Analyses of cell proliferation and apoptosis biomarkers revealed that sulfarotene significantly reduced the expression of Ki67 while increasing Caspase-3 in TRCs compared with ACR, sorafenib and the carrier DMSO (Fig. 1d).

Next the effects of sulfarotene on the growth and formation of subcutaneous xenograft nodes derived from the inoculated Hep3B-TRCs in vivo in BALB/c nude mice were investigated. Both the volumes and weights of the formed Hep3B-TRCs tumor nodes were significantly reduced by sulfarotene administration every other day for a total of 25 days at a concentration of $0.22 \mathrm{mg} / \mathrm{kg}$ or $2.2 \mathrm{mg} / \mathrm{kg}$, compared to $1.8 \mathrm{mg} / \mathrm{kg}$ for ACR and $0.1 \%$ DMSO carrier $(P<$ 


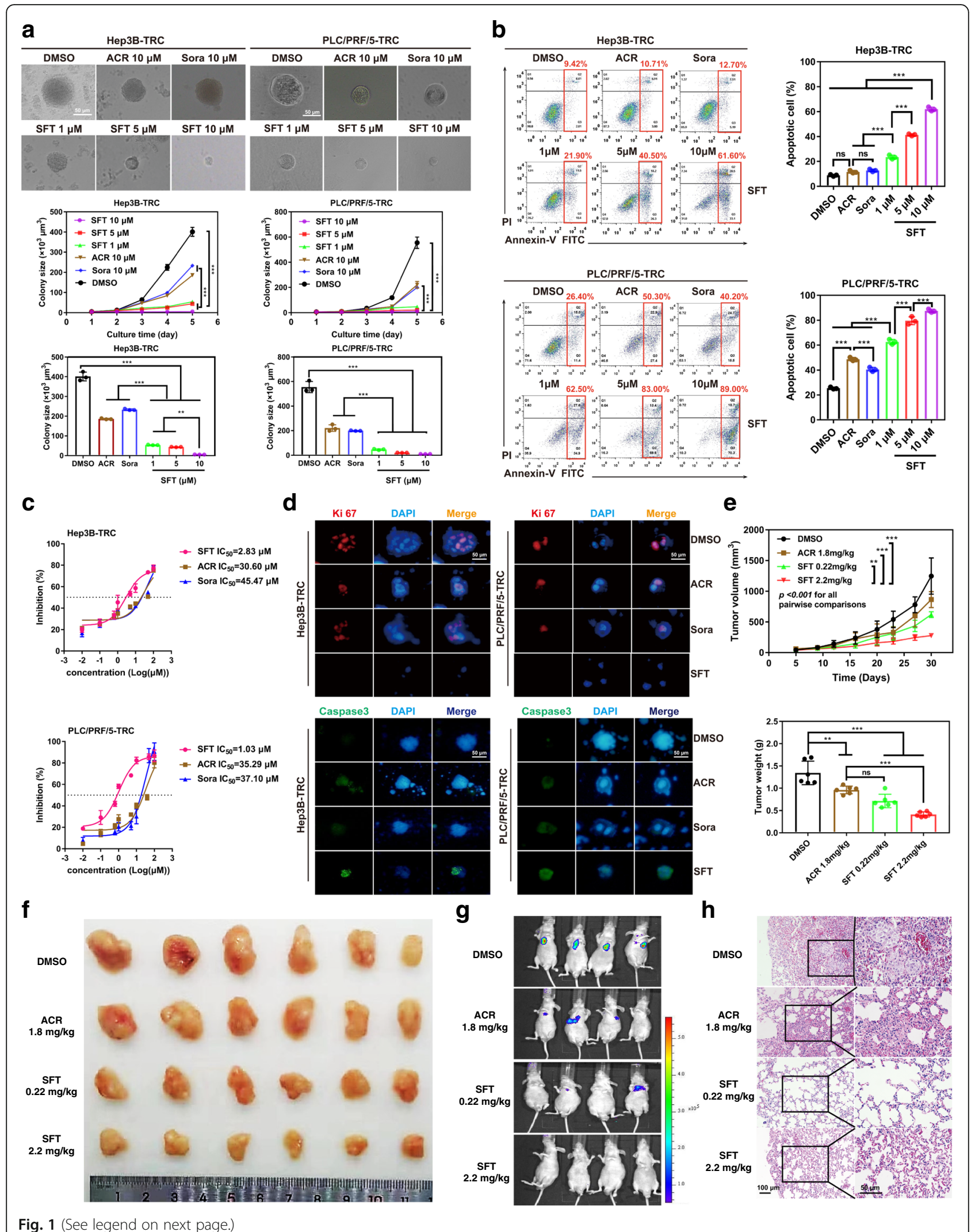


(See figure on previous page.)

Fig. 1 Sulfarotene selectively targets HCC TRCs. a Inhibition of colony spheroid formation in TRCs. Upper panel, the representative images of HCC TRC colony spheroids on day 5 (treatment day 4). Middle panel, quantitative analysis of time-dependent changes of spheroid sizes. Down panel, change of spheroid size on day $5(n=3)$. b Apoptotic effects of sulfarotene at 1.0, 5.0 and $10 \mu \mathrm{M}$ on HCC cell derived TRCs were determined by flow-cytometry with Annexin $\vee$ and propidium iodide (PI) double staining, compared with DMSO (0.1 \% DMSO-containing medium), $10 \mu M$ ACR, and $10 \mu \mathrm{M}$ sorafenib on treatment day 2 (left) $(n=3)$. Right, bar graph presentation for the percentage of apoptotic cells based on flowcytometry analysis. c The $I_{50}$ values of sulfarotene, ACR and sorafenib for HCC TRCs were determined in the CCK8 assay after treatment for $48 \mathrm{~h}$ $(n=3)$. $\mathbf{d}$ Representative immunofluorescence images for the changes of Ki-67 (red) and Caspase-3 (green) in the HCC TRCs in response to treatment with $10 \mu \mathrm{M}$ each of sulfarotene, acyclic retinoid and sorafenib for $48 \mathrm{~h}$ as compared to parental cancer cells. e, $\mathbf{f}$ Inhibitory effects of sulfarotene on growth and xenograft tumor node formation of Hep3B-TRCs subcutaneously transplanted to the flanks of BALB/C nude mice $(n=6)$. Five days after transplantation of TRCs, mice were treated with sulfarotene at 0.22 and $2.2 \mathrm{mg} / \mathrm{kg}$ compared to ACR at $1.8 \mathrm{mg} / \mathrm{kg}$ and the DMSO carrier. $\mathbf{g}, \mathbf{h}$ Inhibition of lung metastasis in mice produced by sulfarotene compared to ACR. Hep3B-TRCs were injected through the tail vein into mice. After 5 days, 0.22 and $2.2 \mathrm{mg} / \mathrm{kg}$ sulfarotene and $1.8 \mathrm{mg} / \mathrm{kg}$ ACR in $0.1 \%$ DMSO (vehicle control) in PBS were injected every 2 days for 25 days. Left, representative bioluminescence images of lung metastasis after treatment for 25 days. Right, HE staining of lung sections with TRC-derived metastasis foci (inset). Tukey's post hoc test. SFT, sulfarotene; sora, sorafenib; ACR, acyclic retinoid. Data are presented as the mean $\pm \mathrm{SD} ;{ }^{*} P<0.05,{ }^{* *} P<0.01,{ }^{* * *} P<0.001$. ns, not significant

0.001) (Fig. 1e, f). Five days after injection of $1 \times 10^{5}$ Hep3B-TRCs through the tail vein, treatment with $0.22 \mathrm{mg} / \mathrm{kg}$ sulfarotene for 25 days reduced the lung metastasis foci by $50 \%$ and $2.2 \mathrm{mg} / \mathrm{kg}$ completely blocked lung metastasis. By contrast, a dose as high as $1.8 \mathrm{mg} / \mathrm{kg}$ of ACR only reduced lung metastasis by up to $25 \%$, and the control of $0.1 \%$ DMSO had no effect on lung metastasis (Fig. $1 \mathrm{~g}, \mathrm{~h}$ ). There were no detectable toxic or necrotic effects on the heart, liver, spleen, lung or kidney tissues after sulfarotene treatment, based on structural morphology of the HE stained sections (Figure S5). Taken together, these data suggest that sulfarotene exhibits significantly better selective therapeutic activity against tumorigenesis from the HCC tumor-repopulating cells than other HCC targeted drugs.

\section{Sulfarotene modulates RARa to confer therapeutic sensitivity to HCC TRCs}

$\mathrm{RA}$ is an important physiological ligand of the nuclear receptors RAR and RXR that when activated exert growth inhibitory effects on various tumors [15]. However, whether sulfarotene that exerts high therapeutic selectivity and efficacy on the TRCs of HCC as described above utilizes a similar RAR- or RXR-dependent mechanism remains unclear. We first performed transcriptome sequencing and found that the mRNA levels of RAR $\alpha$ increased under the action of sulfarotene at $5.0 \mu \mathrm{M}$, but not its homologs RAR $\beta$ and RARY (Fig. 2a). qPCR and western blot analyses revealed that both the mRNA and protein levels of RAR $\alpha$ significantly increased in a dosedependent manner in both Hep3B-TRCs and PLC/ PRF/5-TRCs in response to the treatment with sulfarotene (Fig. 2b, c). Although sulfarotene treatment significantly inhibited colony formation in 3D soft fibrin gels, as well as migration and invasion in transwell culture of TRCs in vitro, pre-treatment with a selective RAR $\alpha$ activation antagonist BMS195614 could significantly reverse these inhibitory effects (Fig. 2d, e). Similar results were obtained with RAR $\alpha$-specific siRNAs, which markedly reduced the mRNA and protein levels of RAR $\alpha$ (Figure S6), accompanied by a reversal of the inhibitory effects imposed by sulfarotene on colony spheroid formation as well as the migration and invasion of TRCs (Figure S7a-c). As expected, immunofluorescence staining and nuclear fractionation studies revealed that sulfarotene treatment promoted the translocation of the activated RAR $\alpha$ from the cytosol into the nucleus (Fig. 2f, g). These results strongly indicate that RAR $\alpha$ is a potential target of sulfarotene in mediating the selective suppression of HCC TRCs.

\section{SOS2 potentially serves as an oncogenic factor in HCC TRCs}

RAR $\alpha$ has been demonstrated to be a key transcriptional regulator of various oncogenes [16, 17]. To understand how RAR $\alpha$ mediates the elevated therapeutic sensitivity of TRCs to sulfarotene (Fig. 1c), we analyzed the downstream target genes associated with RAR $\alpha$ activation and sulfarotene effects by multiple integrative analyses. Multiple comparisons of clusters of the most differentially expressed genes (DEGs) were carried out with data obtained by RNA-Seq in Hep3B, Hep3B-TRCs and L02 cells treated with $0,1.0$ and $5.0 \mu \mathrm{M}$ sulfarotene as well as by ChIP-Seq signals in Hep3B-TRCs treated with 5.0 $\mu \mathrm{M}$ sulfarotene compared to the control $0.1 \%$ DMSO (Fig. 3a, b). The syngeneic DEGs analyses among the 3 groups classified 15,319 union genes of dynamic changes into Venn diagrams and 8 patterns (clusters 1 to 8) with Mfuzz (version 2.50.0) [18] (Fig. 3c, d).

A subsequent integrative analysis on the RNA-Seq clusters (genes from clusters 1,3 and 5) and the ChIP-Seq signals $(M-v a l u e>0.5, \quad P<0.05)$ mapped 


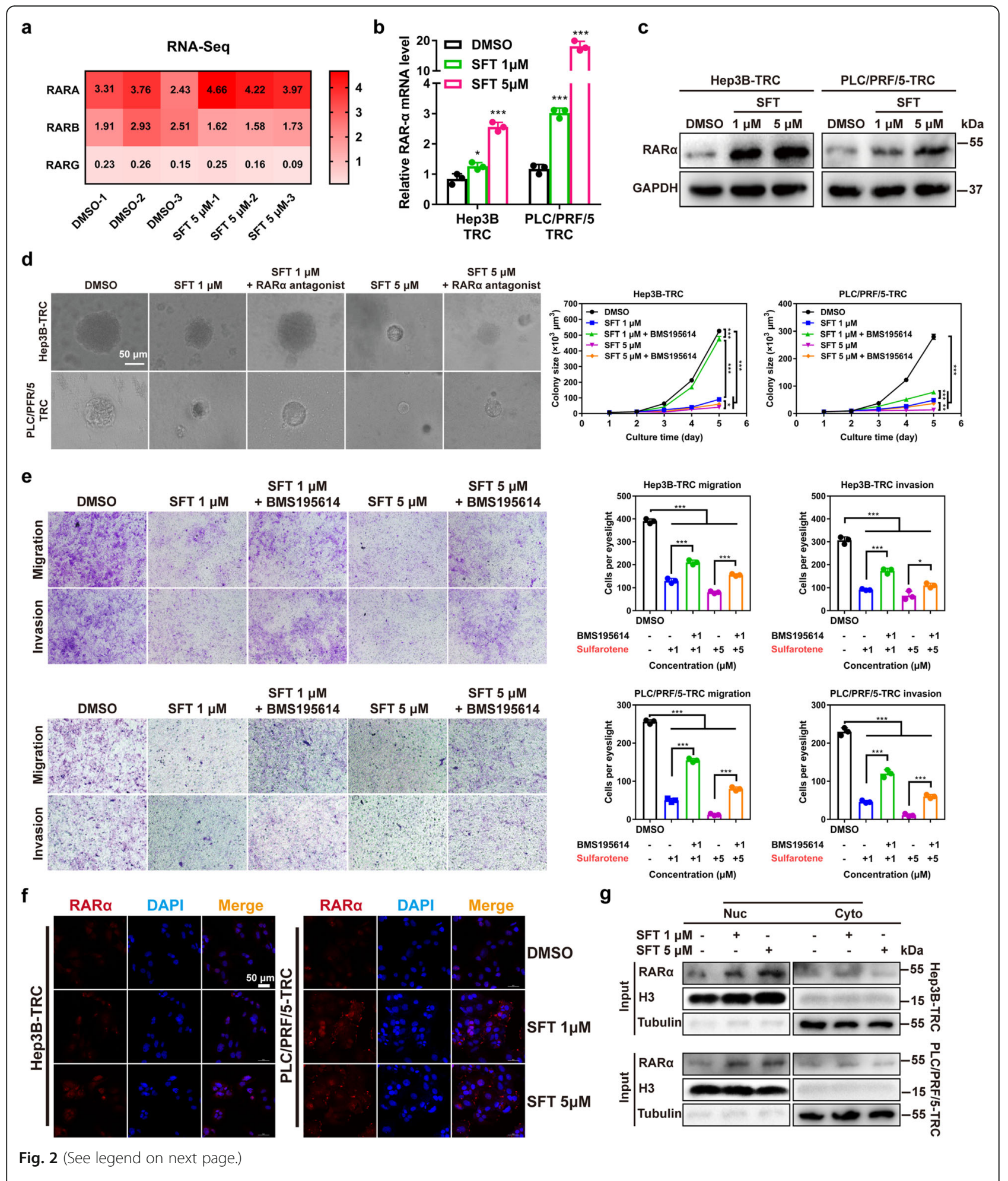


(See figure on previous page.)

Fig. 2 Sulfarotene potentially targets RARa in HCC TRCs. a RNA-Seq analysis revealed that RARa mRNA expression was upregulated in HCC TRCS after sulfarotene treatment compared to that of RARß and RARY. $\mathbf{b}$ Upregulation of RARa mRNA expression in HCC TRCs after sulfarotene treatment. Hep3B-TRCs and PLC/PRF/5-TRCs were treated with sulfarotene at 1.0 and $5.0 \mu \mathrm{M}$ compared to DMSO control for $48 \mathrm{~h}$. The expression levels of RARa relative to the control were normalized to GAPDH $(n=3)$. c Increase of RARa protein levels in Hep3B-TRCs and PLC/PRF/5-TRCs in response to dose-dependent sulfarotene treatment as revealed by western blotting. GAPDH served as the internal reference. $\mathbf{d}$ Effects of sulfarotene and an RARa antagonist on colony spheroid formation from TRCs. Tukey's post hoc test. e Effects of sulfarotene and RARa antagonist on the migration and invasion abilities of TRCs. Tukey's post hoc test. f Representative images of RARa (red) immunofluorescence intensity in Hep3B-TRCs and PLC/PRF/5-TRCs $48 \mathrm{~h}$ after treatment with sulfarotene at 1.0 and $5.0 \mu \mathrm{M}$. DAPI was used to counter-stain the nucleus with blue fluorescence. $\mathbf{g}$ Activation of RARa by sulfarotene as assessed by nuclear translocation. The cytosolic and nuclear fractions Hep3B-TRCs and PLC/ PRF/5-TRCs after sulfarotene treatment for $48 \mathrm{~h}$ were isolated by ultracentrifugation. The RARa protein level in each fraction was analyzed by western blotting. Data are the mean \pm SD of 3 independent experiments; ${ }^{*} P<0.05,{ }^{* *} P<0.01,{ }^{* * *} P<0.001$

402 relevant peaks from which 367 RAR $\alpha$-associated genes were identified (Fig. 3e). Further ranking analyses on the 367 RAR $\alpha$-associated genes according to criteria with 4 priorities, including the degree of neighbor nodes, the total number of involved pathways, the ChIP-Seq peak values and the membership in clusters, identified a list of the top 10 transcriptional target genes of RAR $\alpha$, including SOS2, PTPN11, ATG2B, DCBLD2, SUPT16H, BIRC6, ARFG $E F 2, M Y C B P 2$, TTBK2, and NDST1. Among them, SOS2 appeared to be one of the most relevant genes in determining the highly differential sensitivity of Hep3B-TRCs to sulfarotene in association with RAR $\alpha$ relative to Hep3B cells and L02 cells (Fig. 3f and Supplementary Data 1).

Analyses of single-cell sequencing data of 21 HCC samples obtained from the Gene Expression Omnibus (GEO, GSE149614) (Figure S8a) allowed us to identify a total of 43 clusters that could be classified into 9 cell subsets, including B cells, endothelial cells, hepatocytes, macrophage, monocyte, neurons, NK cells, smooth muscle cells and T cells (Figure S8b). However, none of these cell subsets exhibited clustered distributions with SOS2 gene expression (Figure S8c). Consistent with the above data, we found that SOS2 protein was co-localized with tumor-specific antigen AFP in hepatoma cells but not in other cell types in human HCC tissues (Fig. 3 g).

Furthermore, analyses of the data gathered from human HCC patients by the Cancer Genome Atlas (TCGA) showed that the expression of SOS2 in tumor foci was higher than in the paired adjacent non-tumor tissues (Figure S8d). Analyses of SOS2 expression by qPCR from a cohort of 45 patients, as well as by IHC from a cohort of 237 HCC patients in which 127 had metastasis, revealed that the levels of SOS2 in both the primary and the metastatic tumor foci were higher than in the paired peritumor tissues (Figure S8e-h). Correlation analyses on clinical characteristics and multivariate parameters of 237 HCC patients indicated that SOS2 was an independent prognostic factor associated with both overall survival $(\mathrm{OS})(\mathrm{HR}=1.442, P<0.001)$ and time to recurrence (TTR) $(\mathrm{HR}=1.485, P=0.029)$ (Tables S1 and S2). Survival analyses indicated that patients who were high SOS2 expressors (comprehensive positive score $(\mathrm{CPS})>4$ ) had markedly shorter median overall survival times and times to recurrence than those who were low SOS2 expressors $(\mathrm{CPS} \leq 4)$ (Figure S8i, j) [19]. Functionally, reduction of SOS2 expression by shRNAs significantly impaired the abilities of HCC TRCs to form colony spheroids and of PLC/PRF/5-TRCs to grow subcutaneous xenograft tumor nodes in nude mice (Fig. $3 \mathrm{~h}$, i). Taken together, these results indicated that SOS2 is potentially oncogenic in $\mathrm{HCC}$, and in particular in $\mathrm{HCC}$ TRCs.

\section{Sulfarotene overcomes stemness of HCC via suppressing SOS2}

SOS2 (Son of Sevenless Homolog 2) is an intracellular RAS/Rho guanine nucleotide exchange factor, which promotes the exchange of RAS-GDP to RASGTP thereby activating RAS to allow signal transduction to multiple downstream pathways [20]. To understand the mechanistic relationship between SOS2 and the therapeutic effects of sulfarotene in association with the tumorigenic properties of HCC TRCs, we first found that the protein levels of SOS2 in TRCs were significantly higher than in their parental HCC cells and normal liver cells (Fig. 4a). Then, a significant reduction of SOS2 levels was detected in both types of TRCs in response to sulfarotene treatment in a concentration-dependent manner (Fig. 4b, c). Stable overexpression of SOS2 significantly enhanced colony spheroid formation and subsequent formation of subcutaneous xenograft tumor nodes of these TRCs. By contrast, treatment with sulfarotene at 0.22 and $2.2 \mathrm{mg} / \mathrm{kg}$ markedly repressed both effect parameters promoted by SOS2 overexpression (Fig. 4d-g). Such suppressive effects elicited by sulfarotene were associated with the loss 


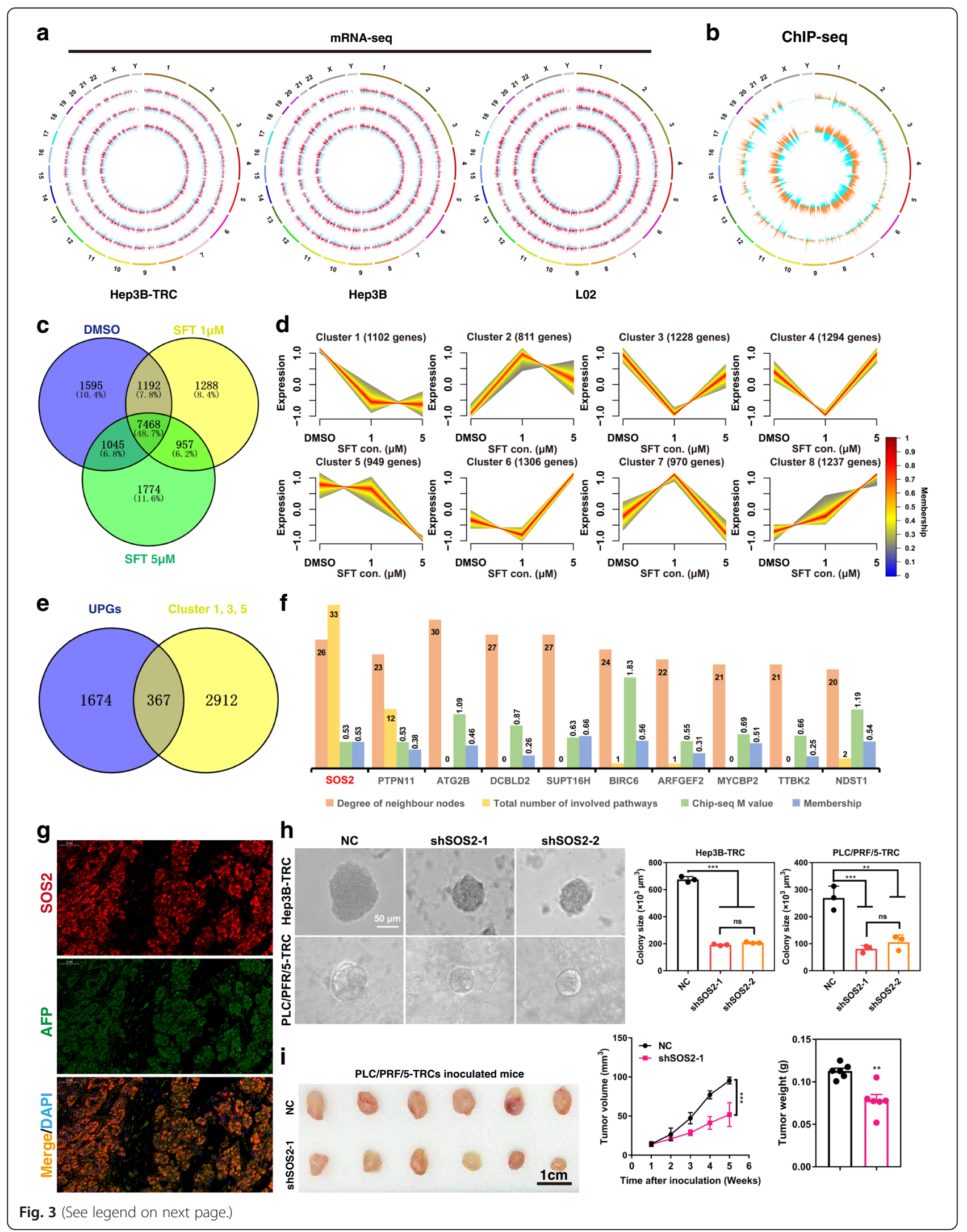




\section{(See figure on previous page.)}

Fig. 3 SOS2 is potentially an oncogenic factor. a A circular diagrams from the most outer circle to the most inner circle represent the log2 fold change value of up-regulated (red) or down-regulated (blue) differentially expressed mRNAs of Hep3B-TRC, Hep3B and L02 cells in 1 or $5 \mu$ M Sulfarotene and DMSO (0.1\% DMSO-containing medium) groups $(n=3, P<0.05)$. b The most outer circle is the chromosome and the middle circle is the promoter that distributes the peak on the chromosome, while the most inner circle is the non-promoter that distributes the peak. The orange sample is $5 \mu \mathrm{M}$ Sulfarotene and the blue sample is DMSO $(n=3, p<0.05)$. The height represents the density of the peak at that location. c Venn diagrams show the number of genes in TRCs in response to treatment with $1 \mu \mathrm{M}$ and $5 \mu \mathrm{M}$ sulfarotene compared to $0.1 \%$ DMSO in the medium. $\mathbf{d}$ Eight dynamic expression change patterns of union genes from the heat map in association with sulfarotene dose escalation were generated by Mfuzz. SFT con., sulfarotene concentration. e Venn diagrams show the number of genes identified as potential RARa-associated targets in TRCs in response to treatment with sulfarotene according to a combined analysis of RNA-Seq and ChIP-Seq data. $\mathbf{f}$ Identification of critical genes clustered in response to sulfarotene and in association with RARa. $\mathbf{g}$ Representative immunofluorescence images for the expression and co-localization of SOS2 (red) and AFP (green) among cells in the HCC tumor tissues. $\mathbf{h}$ Knockdown of SOS2 expression by shRNAs suppressed colony spheroid growth and formation from HCC TRCs cultured in 3D fibrin gels $(n=3)$. NC, negative control shRNA. Tukey's post hoc test. i Knockdown of SOS2 expression suppressed tumor node formation derived from PLC/PRF/5-TRCs in nude mice in vivo, as showed by the corresponding volume and weight of the nodes $(n=6)$. Data are presented as the mean \pm SD; ${ }^{*} P<0.05$, ${ }^{* *} P<0.01$, ${ }^{* * *} P<0.001$. ns, not significant

of both SOS2 and the cell proliferation marker Ki67 and with the increase of apoptotic Caspase-3 (Fig. $4 \mathrm{~h}$ ). These results indicated that sulfarotene potentially reduces SOS2 expression levels to elicit the observed anti-TRC and anti-tumor activity.

\section{Oncogenic SOS2 is transcriptionally targeted by RARa in TRCs of HCC}

To understand the mechanism(s) underlying the anti-tumor effect of sulfarotene through SOS2, we set about determining if RAR $\alpha$ and SOS2 were closely associated in response to and in mediation of sulfarotene treatment as suggested by the data shown above. Treatment with sulfarotene inhibited the expression of SOS2 while the RAR $\alpha$ antagonist BMS195614 rescued the reduction both in Hep3BTRCs and PLC/PRF/5-TRCs (Figure S9a). Overexpression of RAR $\alpha$ directly lowered the expression levels of SOS2 in these TRCs (Figure S9b). Moreover, IHC analyses on the previous xenograft tumors treated with sulfarotene (Fig. 1f) revealed that SOS2 was significantly suppressed while RAR $\alpha$ was substantially elevated upon sulfarotene treatment in comparison with ACR and the negative control DMSO (Figure S10). Nevertheless, upon sulfarotene treatment, RAR $\alpha$ and SOS2 were observed to colocalize in the nucleuses of HCC TRCs, even though the expression of SOS2 decreased (Fig. 5a), indicating an inverse association between SOS2 and RAR $\alpha$.

Based on the Eukaryotic Promoter Database, analysis of the SOS 2 gene promoter region revealed 2 typical RAR $\alpha$ binding elements located at -861 and $641 \mathrm{bp}$, respectively (Fig. 5b), which were confirmed by a luciferase reporter assay showing that RAR $\alpha$ overexpression decreased the luciferase activity driven by the wild-type SOS2 promoter. Interestingly, disruption of these two elements not only failed to rescue but even further reduced the SOS2 expression driven by RAR $\alpha$ overexpression (Fig. 5b), indicating the existence of other RAR $\alpha$ response transcriptional regulatory region(s) in the SOS2 gene locus. Indeed, ChIP-Seq data showed a strong increase in RAR $\alpha$ binding due to sulfarotene treatment in Hep3B-TRCs at +1154 to +1264 bp of the exon 7 locus of the SOS2 gene, while sulfarotene treatment decreased the transcriptional levels and the signals of the surrounding enhancer-like elements marked by H3K27ac of the SOS2 gene (Fig. 5c, d). Subsequent ChIP-PCR analysis indicated that the RAR $\alpha$-binding sites were enriched in both the promoter and exon 7 regions on the SOS2 gene in both TRCs (Fig. 5e, f, Figure S11 and Table S3). 3D structure simulation using the MOE program illustrated a functional binding contact between the critical amino acids (Gly-94, Gly-97, His112, Arg-126, Leu-132, Cys-135 and Glu-160) in the DNA-binding domain of human RAR $\alpha$ and the RAR $\alpha$ response element in exon 7 of the SOS2 gene (Figure S12).

Furthermore, we found that the ChIP-Seq signals for H3K27ac decreased at the SOS2 locus in sulfarotene-treated TRCs. We speculated that the sulfarotene-induced RAR $\alpha$ might also inhibit the super enhancers (SEs) of SOS2 or other critical gene binding sites. By ranking according to increasing H3K27ac enrichment, we identified 1,028 SEs, among which 767 were associated with RAR $\alpha$ (Fig. 5 g). Compared to the DMSO control, RAR $\alpha$ binding was dramatically reduced at SEs by sulfarotene (Fig. $5 \mathrm{~h}$ ). Although no SEs could be found at the SOS2 gene locus, the ChIP-Seq signals for H3K27ac were found to be decreased at the typical enhancers (TEs) in the SOS2 gene and at the SEs of HSPB1 as a control, which has been shown to promote oncogene addiction in many types of cancer including $\mathrm{HCC}$, 


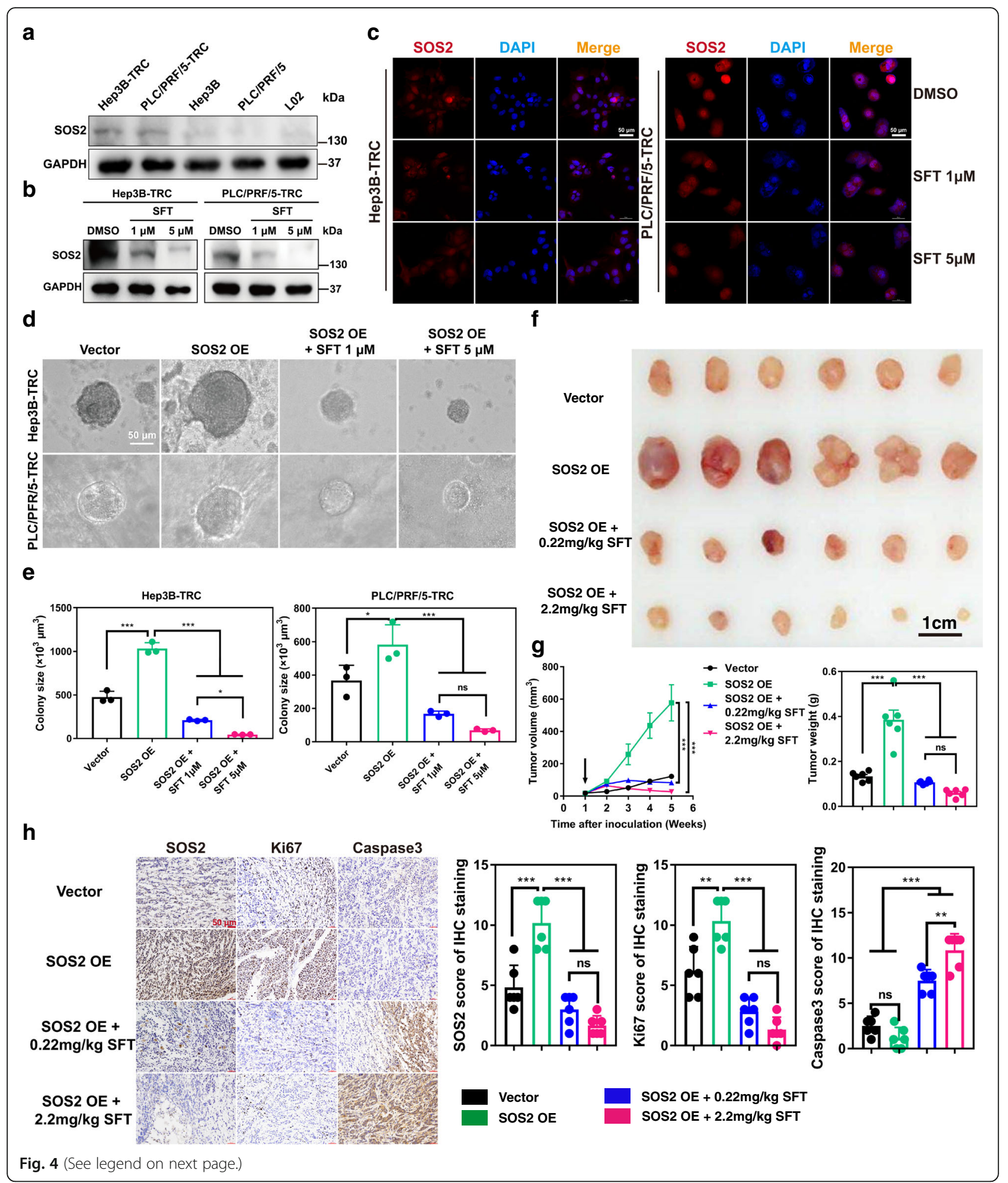


(See figure on previous page.)

Fig. 4 Roles of SOS2 in tumorigenesis and sensitivity to sulfarotene of HCC TRCs. a Higher levels of SOS2 protein were found in Hep3B-TRCs and PLC/PRF/5-TRCs than that in Hep3B, PLC/PRF/5 and L02 cells, as determined by western blotting. b Reduction of SOS2 protein levels in Hep3BTRCs and PLC/PRF/5-TRCs by treatment with sulfarotene at 1.0 and $5.0 \mu \mathrm{M}$ for $48 \mathrm{~h}$, as revealed by western blotting. GAPDH served as the loading control. c Suppression of SOS2 levels by sulfarotene. Representative images for immunofluorescence staining of SOS2 (red) in Hep3B-TRCs and PLC/PRF/5-TRCs cultured in 3D soft fibrin gels after treatment with sulfarotene at 1.0 and $5.0 \mu \mathrm{M}$ for $48 \mathrm{~h}$. Blue, DAPI counterstain of the nucleus. d-e Effects of SOS2 overexpression on colony spheroid formation and sensitivity to sulfarotene of HCC TRCs. Hep3B-TRCS and PLC/PRF/5TRCs stably overexpressing SOS2 were treated with sulfarotene at concentrations of 1.0 and $5.0 \mu \mathrm{M}$. After $48 \mathrm{~h}$, the size of colony spheroids was determined in $\mu^{3}$. $\mathbf{f}-\mathbf{g}$ Effects of SOS2 overexpression on xenograft tumor node formation and sensitivity to sulfarotene of HCC TRCs. Spheroids of PLC/PRF/5-TRCs stably overexpressing SOS2 were allowed to form in 3D soft fibrin gels for 1 week, and then inoculated subcutaneously into the flanks of nude mice. A bolus injection of sulfarotene at 0.22 or $2.2 \mathrm{mg} / \mathrm{kg}$ was given i.v. to nude mice once every two days. After 4 weeks, tumor nodes (e) were dissected from the mice and tumor volumes and weights $(\mathbf{f})$ were measured $(n=6)$. Down arrow indicates the start of sulfarotene treatment. $\mathbf{h}$ Effects of SOS2 overexpression on the proliferation and apoptosis of tumor nodes and sensitivity to sulfarotene of HCC TRCs. Left, representative IHC images for the expression levels of SOS2, Ki-67 and Caspase-3 in sections of xenograft tumor nodes (e) from PLC/ PRF/5-TRCs overexpressing SOS2 or after sulfarotene treatment compared to controls. Right, statistical analysis of the IHC scores in each group $(n=6)$. Tukey's post hoc test. Data are presented as the mean \pm SD of 3 independent experiments; ${ }^{*} P<0.05,{ }^{* *} P<0.01,{ }^{* * *} P<0.001$. ns, not significant

whereas RAR $\alpha$ increased the binding to such loci in SOS 2 and HSPB1 in sulfarotene-treated TRCs (Fig. 5i, j). Taken together, these results suggest that SOS2 is a direct transcriptional target of RAR $\alpha$ that is targeted by sulfarotene.

\section{Sulfarotene inhibits HCC TRCs by suppressing SOS2-RAS associated signaling pathways}

As SOS2 serves as a signaling center in association with RAS [20], we analyzed the possible KEGG pathways associated with SOS2-RAS activation in these HCC TRCs. Analyses of the dynamic changes of SOS2 and its 220 neighboring genes identified by RNA-Seq in response to sulfarotene treatment, according to significant Pearson correlation coefficients (PCCs), allowed us to abstract and construct a SOS2-centered participating pathway network (Fig. 6a and Supplementary Data 2). Most of the identified pathways are involved in RTK-mediated RAS signaling and downstream pathways, including PI3K/AKT and MEK/ERK (Fig. 6b). Using a human phosphoRTK array, we showed that the phosphorylation levels of several RTKs were significantly elevated compared with those of the parental cancer cells (Fig. 6c). Among the commonly associated pathway mediators downstream of these RTKs, the levels of GTP-RAS, p-MEK1/2, p-ERK1/2 and p-AKT were significantly elevated (Figure S13a, b). Sulfarotene treatment for $48 \mathrm{~h}$ markedly reduced the levels of both SOS2 and GTP-RAS while significantly increasing RAR $\alpha$, which were companied by decreases in $\mathrm{p}$ MEK1/2, p-ERK1/2 and p-AKT in a concentrationdependent manner (Fig. 6d e). Of note, overexpression of SOS2 significantly increased the activation of these SOS2-RAS associated pathways while sulfarotene treatment or SOS2 knockdown effectively reversed these changes (Figure S13c-f).

\section{Sulfarotene overcomes sorafenib resistance via SOS2-RAS pathway suppression}

As mentioned previously, both types of TRCs exhibited strong sorafenib resistance. We found that the phosphorylation levels of several known targets of sorafenib including PDGFR $\alpha$, PDGFR $\beta$ and VEGFR3 were downregulated in PLC/PRF/5-TRCs, indicating a possible mechanism of sorafenib resistance, while the increased phosphorylation levels in the increased number of other RTKs might underlie sorafenib resistance in Hep3B-TRCs (Figure S14). Furthermore, a combination of sorafenib and sulfarotene effectively abolished the inability of sorafenib to suppress the active levels of the SOS2-RAS associated mediators in these HCC TRCs (Fig. 7a, b). In addition, we calculated the combination index (CI) of sulfarotene and sorafenib, and found that the two drugs have a synergistic effect $(\mathrm{CI}=0.51$ of Hep3B-TRC and $\mathrm{CI}=$ 0.73 of PLC/PRF/5-TRC). Further, SOS2, as the target of sulfarotene, knockdown combined with sorafenib exhibited the lower IC50 values of $7.68 \mu \mathrm{M}$ and $14.39 \mu \mathrm{M}$ of the selected Hep3B-TRCs and PLC/PRF/5-TRCs after $48 \mathrm{~h}$ treatment compared with sorafenib alone treated for HCC TRCs, which is similar to the effect of sorafenib treated for HCC cell lines (Figure S15). Consistent with the inhibition of such growth-promoting and apoptosissuppressing pathways, the orthotopic xenograft tumors from PLC/PRF/5-TRCs inoculums succumbed to the combination regimen in vivo accompanied by the loss of sorafenib resistance, the decreases in pERK1/2 and p-AKT, and the increase in Caspase-3 (Fig. 7c-g). Collectively, the results suggested that 
a

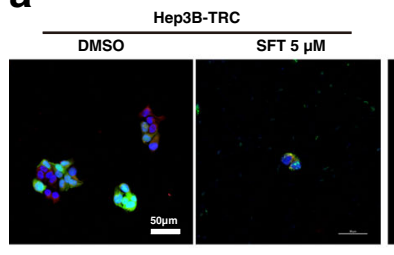

C

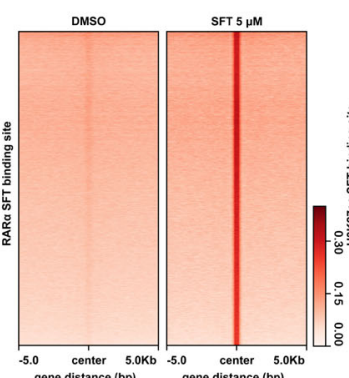

d

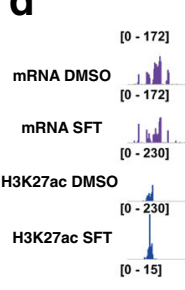

RAR $\alpha$ DMSO

$\operatorname{RAR} \alpha \mathrm{SFT}$

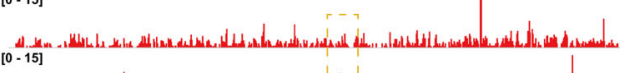

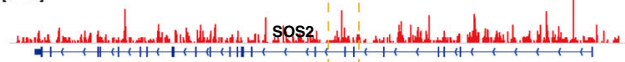

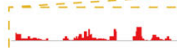

$\operatorname{Exon}_{1}$

g

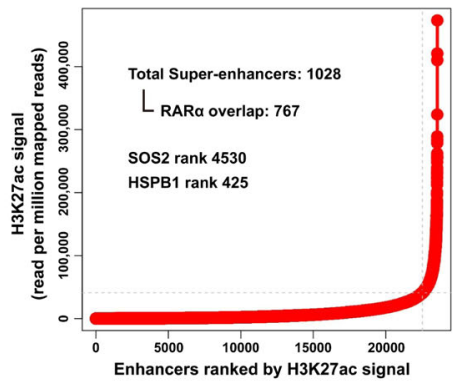

i
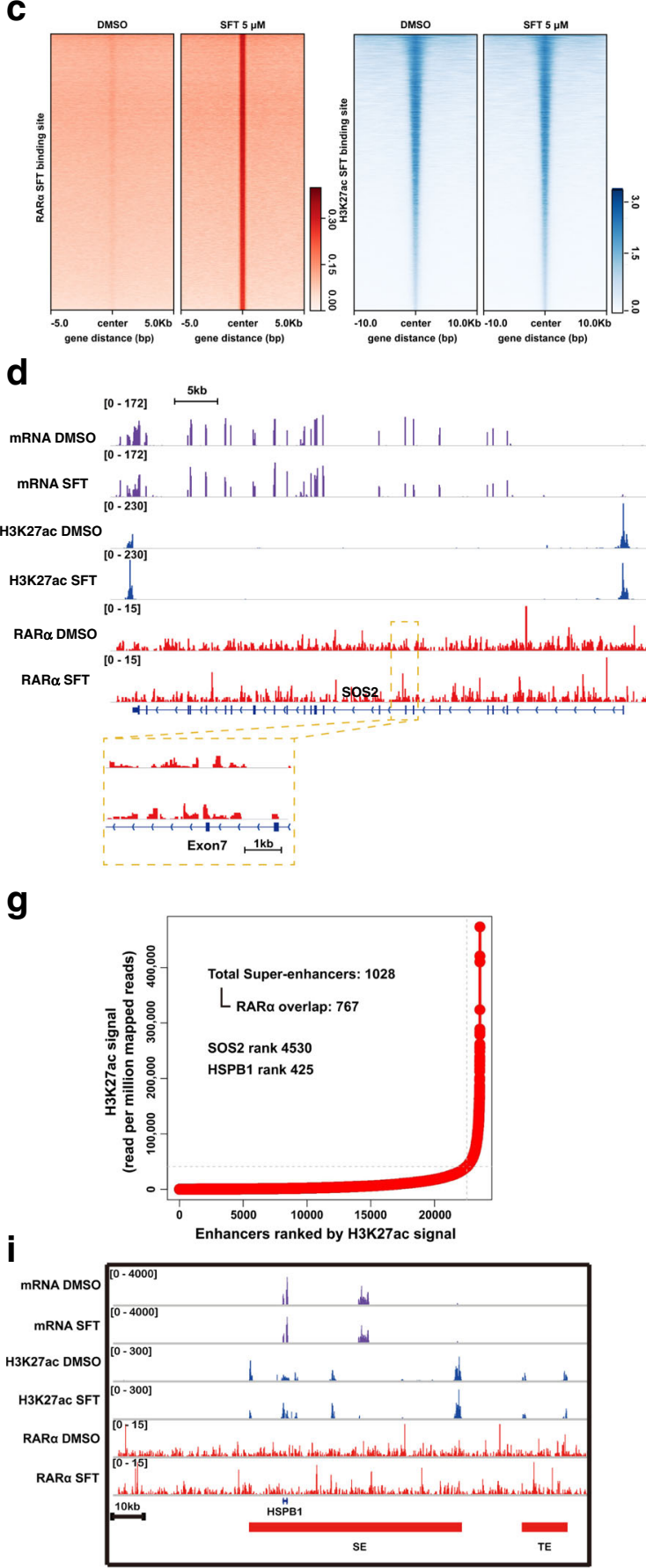

b

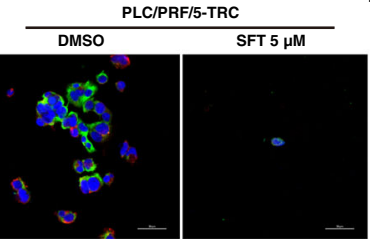

e
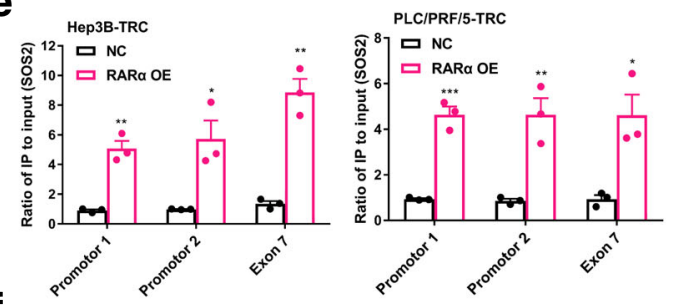

f

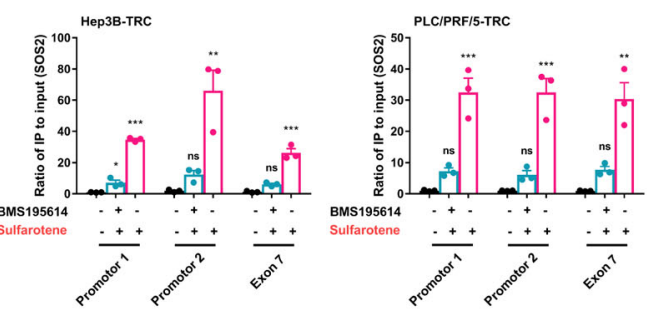

h

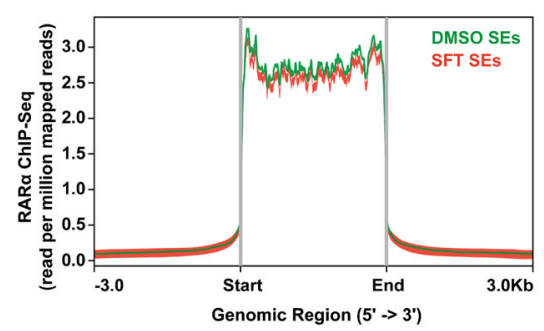

j

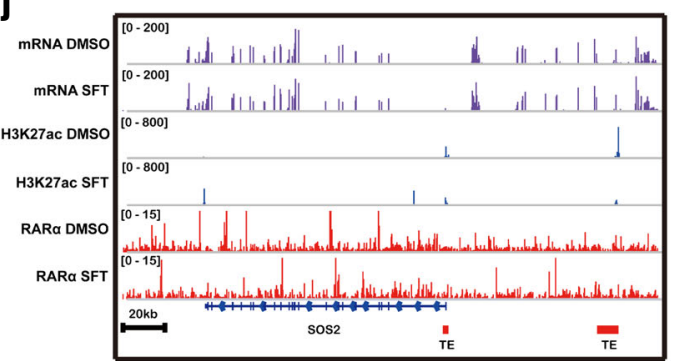

Fig. 5 (See legend on next page.) 
(See figure on previous page.)

Fig. 5 Sulfarotene inhibits SOS2 transcription via RARa. a Immunofluorescence showed that RARa (red) colocalized with SOS2 (green) in the nucleus of HCC TRCS treated with sulfarotene. DAPI: blue. b Map of the SOS2 5 ' region from the +1 transcription starting site. Amplicon 1 and 2 , regions of interest within the SOS2 promoter in luciferase reporter experiments. Amplicons 3, region of interest in the exon 7 locus as revealed in ChIP-PCR experiments using anti-RARa antibody. $\mathbf{c}$ Heat map of RARa and H3K27ac ChIP-Seq signals in Hep3B-TRCs in response to sulfarotene treatment as compared to DMSO. Each row shows $\pm 5 \mathrm{~kb}$ centered on the RARa peak. The ChIP-Seq signal was depicted by color scaled intensity. d Gene tracks of ChIP-Seq signals for RNA-Seq, H3K27Ac and RARa signals around the SOS2 loci in Hep3B-TRCs after treatment with $5.0 \mu M$ sulfarotene. ChIP-Seq and RNA-Seq signals were visualized with the Integrated Genome Viewer (IGV). e-f Interactions of RARa with 2 promoter elements and 1 element on exon 7 of SOS2 gene were determined by ChIP-qPCR analysis with anti-RARa antibody and rat lgG as controls. Hep3B-TRCs and PLC/PRF/5-TRCs with stable overexpression of RARa (e) or treated with $5.0 \mu \mathrm{M}$ sulfarotene in the presence or absence of BMS195614 (f) for $24 \mathrm{~h}$ were cultured for 5 more days, then ChIP-qPCR analysis was performed as described. $\mathbf{g}$ The H3K27ac signals indicated enhancers in Hep3B-TRCs treated with sulfarotene relative to DMSO. The super enhancer (SE) zone is illustrated by the dashed lines. $\mathbf{h}$ Metagene representations of RARa ChIP-Seq signals in units of read count per million mapped reads at a meta composite of SEs in Hep3B-TRCs treated with $5.0 \mu \mathrm{M}$ sulfarotene compared to DMSO. i-j Gene tracks of ChIP-Seq signals for RNA-seq, H3K27Ac and RARa signals around the HSPB1 and SOS2 loci in Hep3B-TRCs treated with $5.0 \mu \mathrm{M}$ sulfarotene compared to DMSO. Red bars show the sulfarotene-specific SEs and the typical enhancers (TES)

sulfarotene effectively abolished the activation and oncogenicity of SOS2-RAS associated signaling pathways thus inhibiting the tumorigenicity and drug resistance of HCC TRCs.

\section{Sulfarotene inhibits tumor progression of HCC PDXs with high SOS2 expression}

To determine potential therapeutic efficacy of sulfarotene on HCC, we established PDX models in NOG mice (nonobese diabetic mice with severe combined immunodeficiency) with freshly resected specimens from HCC patients. The HCC specimens were divided into one group with high expression levels $(\mathrm{CPS}>4)$ and the other with low expression levels for SOS2 based on the normalized intensity of IHC stains (Fig. 8a). We found that the volume and weight of tumor nodes derived from PDXs of high SOS2 expressors were larger than that of the low SOS2 expressors, suggesting that the tumor nodes of high SOS2 expressors retained the characteristics of the HCC TRCs (Fig. 8b, c). After treatment with sulfarotene at $0,0.22$ and $2.2 \mathrm{mg} / \mathrm{kg}$ by i.v. injection once every 2 days for 21 days, both the volume and weight of tumor nodes of high SOS2 expressors were markedly suppressed by both the low and high dose treatments compared to that of the low SOS2 expressors and the DMSO control group (Fig. 8d-f). Sulfarotene at $2.2 \mathrm{mg} / \mathrm{kg}$ imposed a as high as $90 \%$ reduction in the volumes of tumor nodes that originated from PDXs with high SOS2 expression compared to only a $52 \%$ reduction in those with low SOS2 expression, demonstrating a predominant role of SOS2 in determining the malignant behavior and the high sensitivity to sulfarotene of HCC TRCs. Consistent with the aforementioned changes in key SOS2-RAS associated pathway mediators, IHC staining of tumor node sections revealed that the levels of SOS2, Ki67 and SOS2-RAS associated downstream mediators (p-MEK1/2, p-ERK1/2 and p-AKT) were remarkably inhibited by sulfarotene in the high SOS2 expressors (Fig. 8 g, h). These results suggested that sulfarotene selectively and significantly inhibited human HCC PDX tumors predominantly by blocking the SOS2-RAS nexus and associated signaling pathways.

\section{Discussion}

Despite significant progress being made in the development of novel therapies, HCC remains among the most recurrent, metastatic, and thus lethal malignancies worldwide [21]. The notable self-renewal ability and high tumorigenicity of resident CSCs have been thought to be responsible for the observed high rate of recurrence, acquired drug resistance, poor prognosis, and treatment failure $[6,7,22]$. Historically, both the natural and synthetic trans-RAs have been used to treat cancers of the hemopoietic system, in particular leukemia, by targeting the nuclear receptor RAR-RXR system, however, the efficacy remains poor for solid tumors due to drug resistance, poor aqueous solubility, poor accessibility, and a short half-life [23]. Only one RA derivative, the acyclic retinoid with a prolonged half-life, has been used to treat recurrent $\mathrm{HCV}$-related solid liver cancer after curative therapy by targeting oncogenic $M Y C N$, which transcriptionally regulates EPCAM, a biomarker of HCC CSCs [24], in addition to RAR-RXR [13, 25, 26]. Much effort has led to the recent development of a new class of synthetic RA-like compound, sulfarotene (also called WYC-209), which in one study exhibited high therapeutic activity against malignant melanoma with little toxicity, which is another type of drug-resistant cancer [8]. Furthermore, a substantial portion of patients with advanced HCC also 

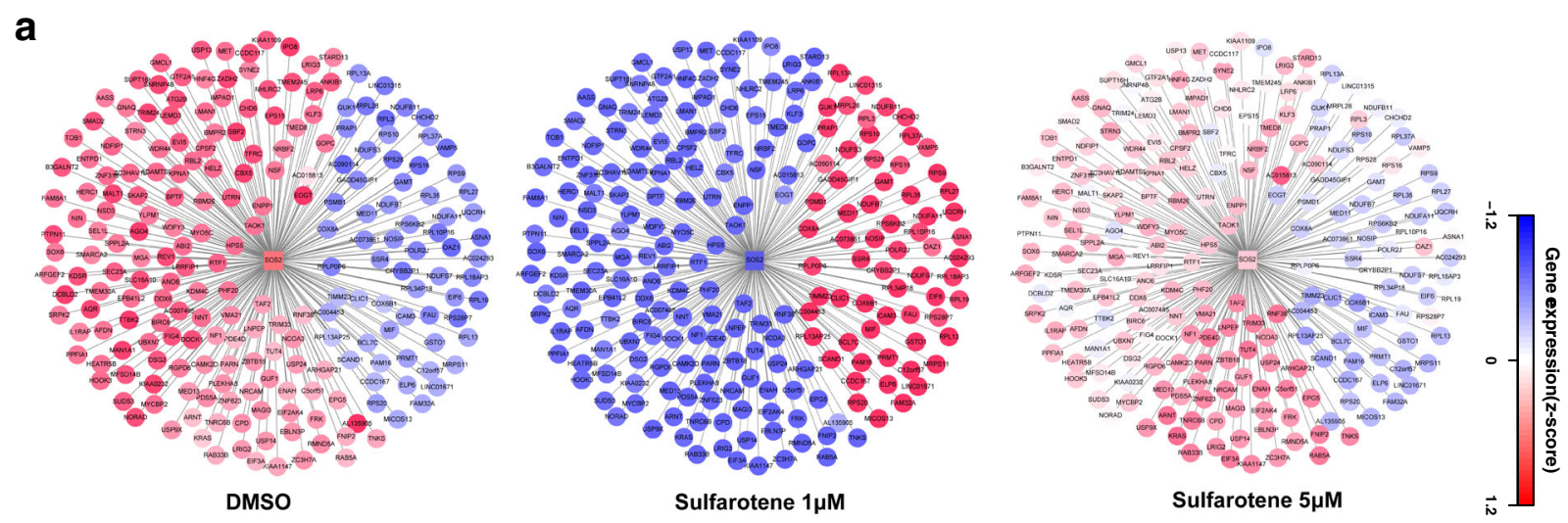

b

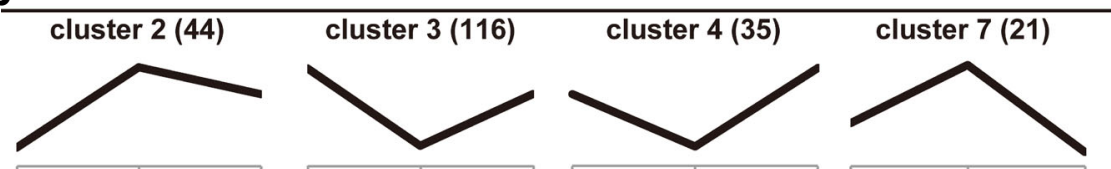

DMSO SFT $1 \mu \mathrm{M}$ SFT $5 \mu \mathrm{M}$ DMSO SFT $1 \mu \mathrm{M}$ SFT $5 \mu \mathrm{M}$ DMSO SFT $1 \mu \mathrm{M}$ SFT $5 \mu \mathrm{M}$ DMSO SFT $1 \mu \mathrm{M}$ SFT $5 \mu \mathrm{M}$
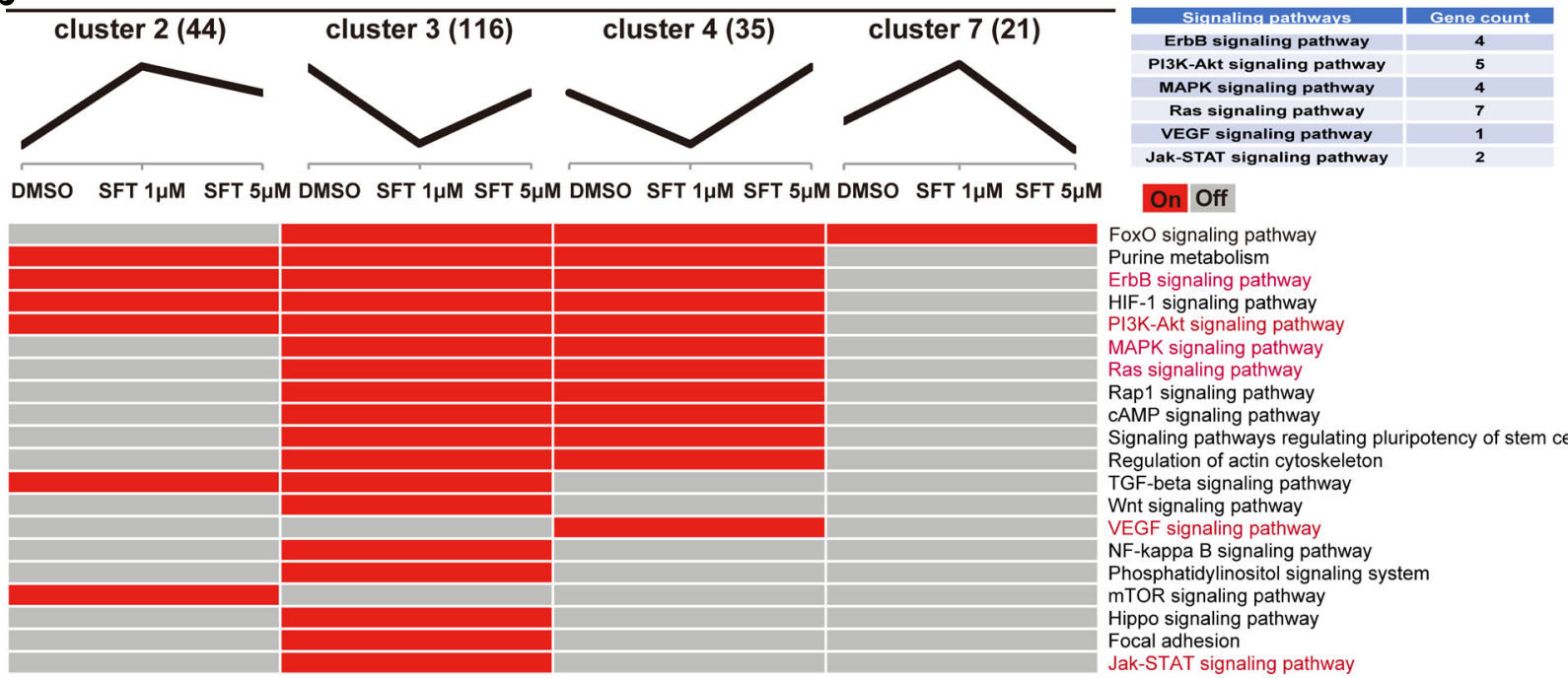

\section{On off}

FoxO signaling pathway

FoxO signaling pat
Purine metabolism

ErbB signaling pathway

HIF-1 signaling pathway

PI3K-Akt signaling pathway

MAPK signaling pathway

Ras signaling pathway

Rap1 signaling pathway

cAMP signaling pathway

Signaling pathways regulating pluripotency of stem cells

Regulation of actin cytoskeleton

TGF-beta signaling pathway

Wnt signaling pathway

VEGF signaling pathway

NF-kappa B signaling pathway

Phosphatidylinositol signaling system

mTOR signaling pathway

Hippo signaling pathway

Focal adhesion
Jak-STAT signaling pathway

C

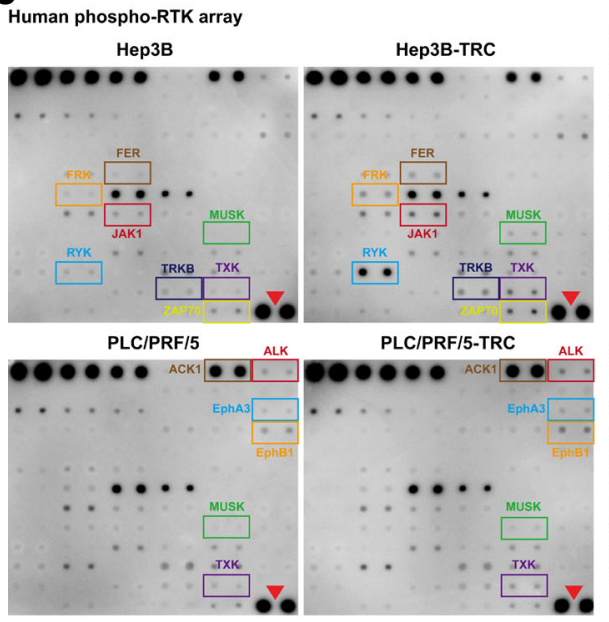

$\checkmark$ Reference Points

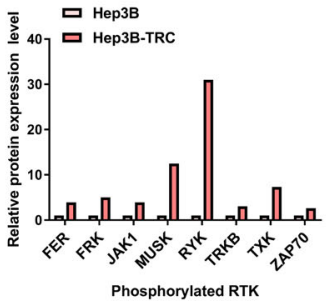

Phosphorylated RTK

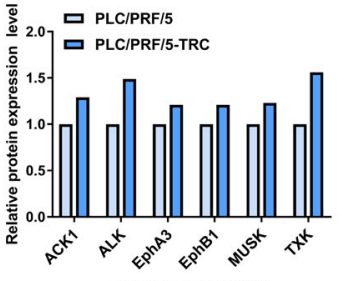

Phosphorylated RTK d

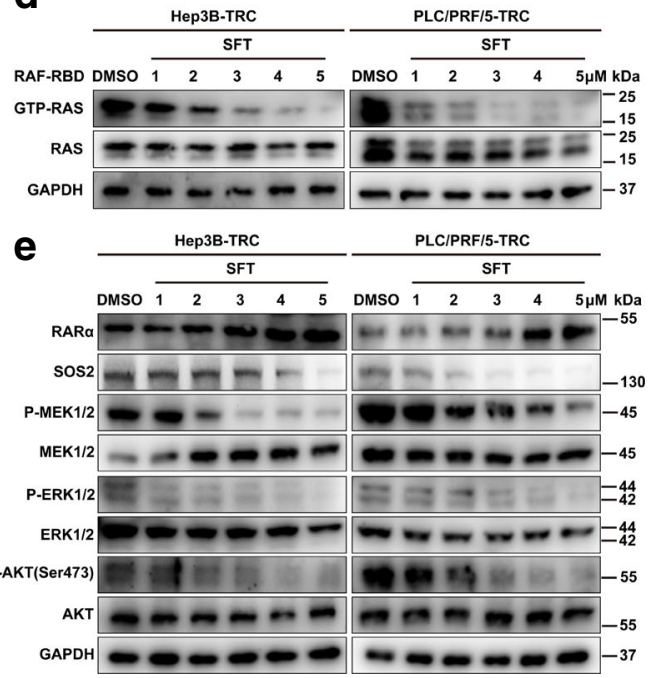

Fig. 6 (See legend on next page.) 
(See figure on previous page.)

Fig. 6 Sulfarotene targets the SOS2-RAS signal nexus. a Dynamic expression changes in SOS2 and associated neighboring genes in response to sulfarotene treatment. Expression changes of SOS2 and its 220 related genes in Hep3B-TRCs after treatment with sulfarotene at 1 and $5 \mu$ M for 2 days were revealed by RNA-Seq analysis $(|\mathrm{PCCS}|>0.9, P<0.05)$ and presented as a network diagram. $\mathbf{b}$ Heat map presentation of SOS2-associated KEGG pathways in response to sulfarotene treatment abstracted from 4 differential, dynamic change patterns of clusters 2, 3, 4 and 7. c Upregulation of phosphorylation of several types of RTKs in HCC TRCS as detected by human phospho-RTK arrays compared to that in parental HCC cancer cells. Left, representative real-time intensity images of the phospho-RTK arrays. Right, summary of the upregulated phospho-RTKs in bar graphs. d-e Dose-dependent inhibition of GTP-RAS, SOS2 and associated PI3K/AKT and MEK/ERK pathway mediators by sulfarotene in association with the promotion of RARa in the HCC TRCs. Hep3B-TRCs and PLC/PRF5-TRCs were treat with sulfarotene at concentrations of 1.0 to $5.0 \mu \mathrm{M}$ for $48 \mathrm{~h}$, and cell lysates were subjected to western blot analyses for the respective proteins as indicated

exhibits resistance to another widely used multikinase inhibitor sorafenib. In this endeavor, we first adapted a simple, recently developed mechanical approach to isolate CSC-like tumor-repopulating cells in 3D soft fibrin gels [6] from human HCC cell lines. We showed that the isolated TRCs recapitulated the tumorigenic and drug-resistant characteristics of CSCs while sulfarotene effectively and selectively suppressed these features in vitro and aborted the growth and formation of xenograft and PDX tumors and associated lung metastasis derived from TRCs inoculums in vivo, without notable side effects compared to ACR and sorafenib.

As a new class of structural analog of RA that serves as an agonist of RAR or RXR, sulfarotene indeed activates RAR $\alpha$, which is in agreement with a previous report [8]. Analyses of the dynamic, differential changes in expression of genes in response to sulfarotene treatment and of the neighboring gene network functionally associated with RAR $\alpha$ activation, revealed SOS2 as a critical player in mediating the therapeutic effects of sulfarotene. Furthermore, we found that SOS2 is a direct target of RAR $\alpha$ as a transcription factor, with several typical RAR response elements to which RAR $\alpha$ binds to repress expression. Like the family member SOS1, SOS2 belongs to the GEF family that promotes the exchange of RAS-GDP to RAS-GTP, thereby activating RAS, leading to activation of an array of downstream signaling pathways, notably the PI3K/ AKT and MEK/ERK pathways, that promote cell growth, survival and migration [27]. Aberrant signaling of these pathways is associated with many types of cancers [28-30]. SOS1 was identified as a predictive gene for HCC prognosis in association with RAS [31], and a small molecular inhibitor BAY-293 that disrupts SOS1-KRAS interaction blocked RAS activation, leading to potent antiproliferative effects [32]. Deficiency of SOS1 and SOS2 prevented malignant progression of chemicalinduced skin papilloma [33]. In the present study, we found that both human HCC tumor foci and the selected TRCs were among the high expressors for SOS2, but not for SOS1, which remained low and unchanged.

Of importance, we observed that the elevated SOS2 levels or RAS activation in the selected TRCs was in accordance with increased activation of several upstream RTKs as well as downstream MEK/ ERK and PI3K/AKT pathways while loss of SOS2 after sulfarotene treatment blocked the accumulation of RAS-GTP and activation of associated pathways, highlighting SOS2-RAS as a central oncogenic nexus. Apart from the aforementioned possible mechanisms, this may also explain why HCC TRCs are resistant to sorafenib while sulfarotene or a combination of sorafenib with sulfarotene effectively overcomes the resistance. Although mutations in RAS were not frequently detected in HCC, overexpression or overactivation of RTKs upstream of RAS can be prominent [34]. In addition to CSCs evolution resistance to sorafenib, there are also studies that have found that epithelial-mesenchymal transition (EMT) transition in tumor microenvironment led to sorafenib resistance. As the core factor of EMT, Snail, miR-182-5p/lncRNA-POIR and ZNF703 are highly expressed in sorafenib resistance cells, while Snail [35], miR-182-5p/lncRNA-POIR [36] and ZNF703 [37] knockdown could restore the sensitivity to sorafenib. Therefore, Regorafenib reversed sorafenib resistance by inhibiting ERK and STAT3, and subsequently downregulating Snail and EMT [38]. Moreover, dysregulated metabolism could be associated with increased sorafenib resistance in HCC [39]. Sorafenib also promoted to export glucose absorption and lactic acid [40]. PFKFB3 [41], HK2 [42], and PKM2 [43], as the key enzymes of glycolysis, have been shown to be overexpressed in sorafenib resistant HCC cell lines to increase glycolysis flux, which were silenced for contributing to synergetic effect with sorafenib. Compared to normal HCC cells, sorafenib resistant CSCs have higher rates of glucose consumption and lactate production, which are highly dependent on glycolysis [44- 


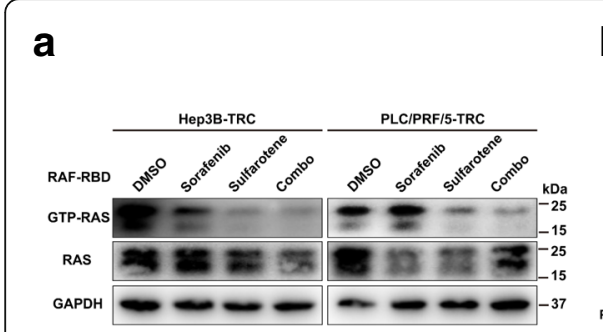

d

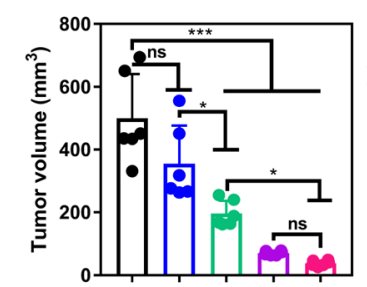

DMSO
SFT $2.2 \mathrm{mg} / \mathrm{kg}$

f
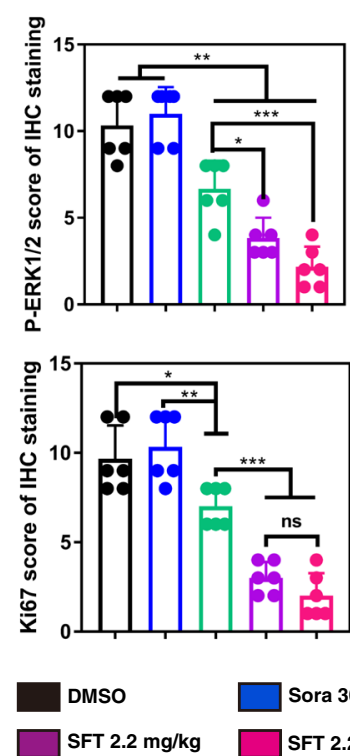

SFT $2.2 \mathrm{mg} / \mathrm{kg}$ + Sora $30 \mathrm{mg} / \mathrm{kg}$ b

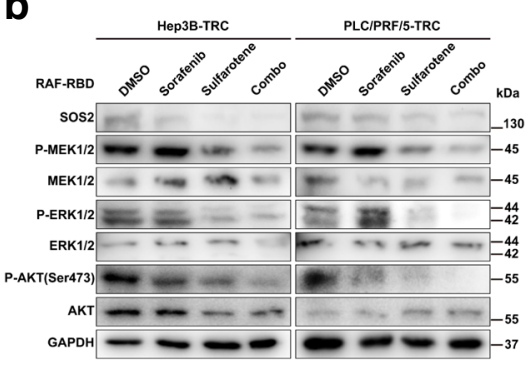

C

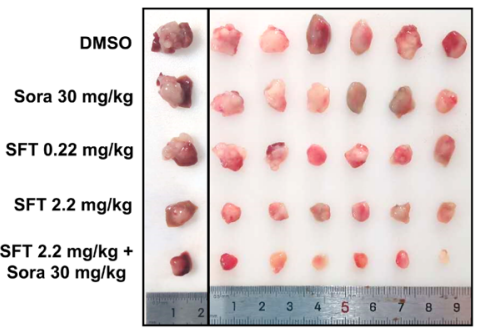

e
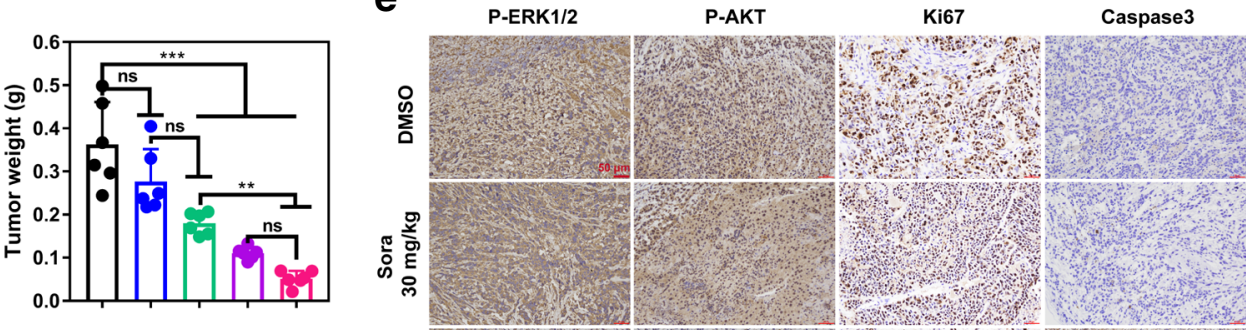

范寉
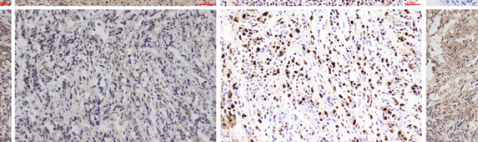

$0.22 \mathrm{mg} / \mathrm{kg}$ क
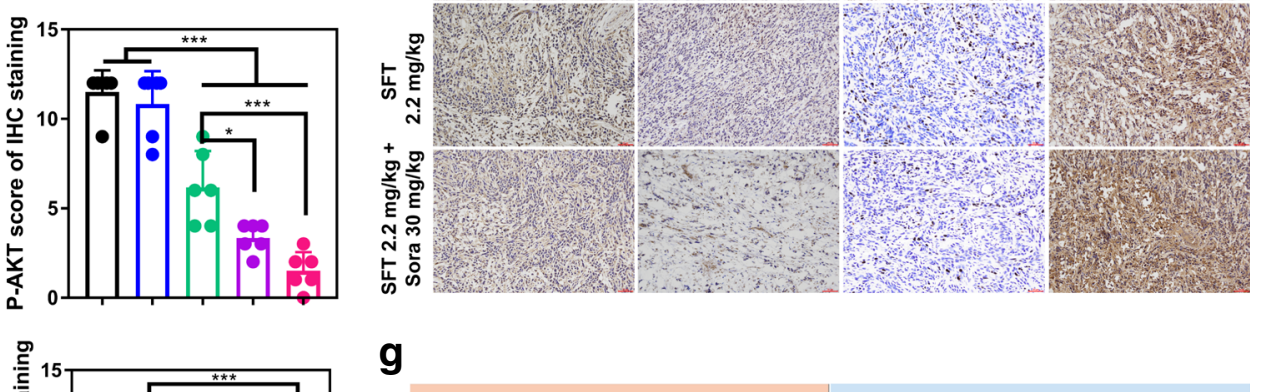

g

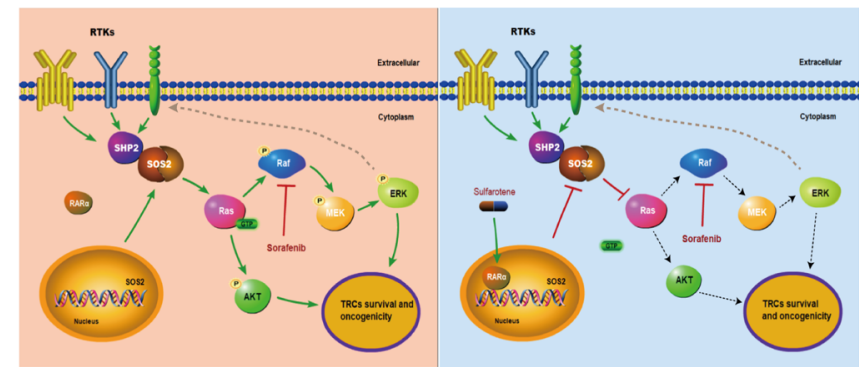

Fig. 7 Sulfarotene inhibits SOS2-RAS nexus and reverses sorafenib resistance. a Immunoblots of whole cell lysates or RAF-RBD precipitated lysates from HCC TRCs after treatment with $5 \mu \mathrm{M}$ sulfarotene, $10 \mu \mathrm{M}$ sorafenib or a combination of both drugs for $48 \mathrm{~h}$. $\mathbf{b}$ Effects of sulfarotene on SOS2 and RAS associated pathways compared to sorafenib. HCC TRCs were treated with sulfarotene, sorafenib or their combination for $48 \mathrm{~h}$ as in (a). Whole cell lysates were then used to assess changes in SOS2, p-MEK1/2, p-ERK1/2 and p-AKT (Ser473) relative to their corresponding total protein levels by western blotting. c-d Effects of sulfarotene on xenograft tumor formation of the selected HCC TRCs compared to sorafenib. $1 \times 10^{5}$ PLC/ PRF/5-TRCs were inoculated subcutaneously to the flanks in nude mice. 7 days later, 0.22 and 2.2 mg/ $\mathrm{kg}$ sulfarotene, $30 \mathrm{mg} / \mathrm{kg}$ sorafenib or a combination of $2.2 \mathrm{mg} / \mathrm{kg}$ sulfarotene and $30 \mathrm{mg} / \mathrm{kg}$ sorafenib was injected every two days for 25 days. Sulfarotene was injected intraperitoneally while sorafenib was administered by oral intra-gastric gavage. The volume and weight (d) of the derived orthotopic xenograft tumor nodes (c) as indicated were measured. e-f Representative $\mathrm{IHC}$ images of the sections (e) from the orthotopic tumor nodes (c) indicate differential changes in the levels of p-ERK1/2, p-AKT, Ki-67 and Caspase-3 in response to treatment with 0.22 and $2.2 \mathrm{mg} / \mathrm{kg}$ sulfarotene, $30 \mathrm{mg} / \mathrm{kg}$ sorafenib, or a combination of $2.2 \mathrm{mg} / \mathrm{kg}$ sulfarotene and $30 \mathrm{mg} / \mathrm{kg}$ sorafenib. Bar graphs (f) show the IHC score \pm SD for each group from 3 independent experiments. Tukey's post hoc test. ${ }^{* * *} P<0.001$. ns, not significant. $\mathbf{g}$ Schema depicting the mechanism by which sulfarotene targets the RARSOS2-RAS signal axis to inhibit cancer cell growth and overcome drug resistance 


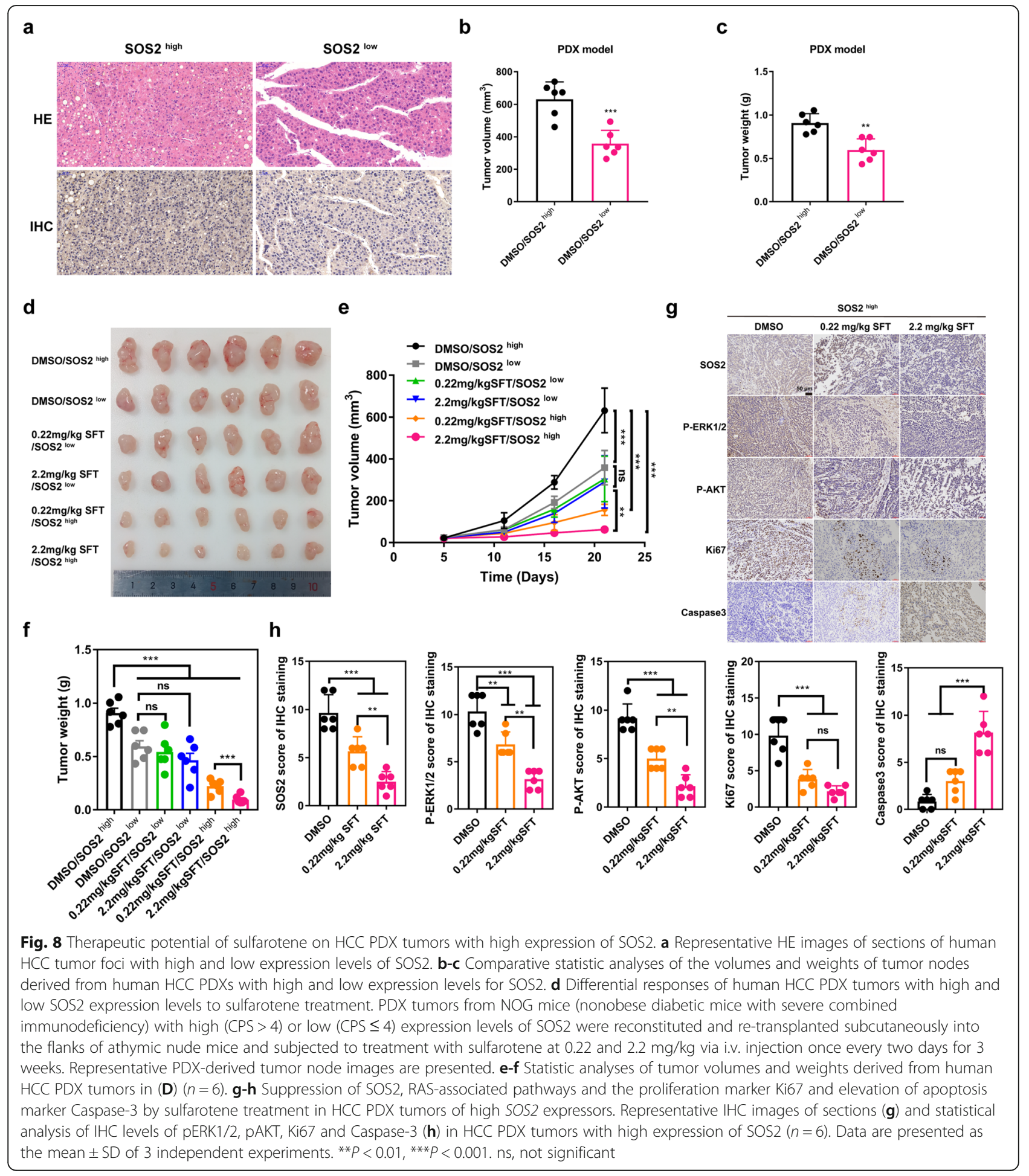

46]. In our study, the rates of glucose consumption and lactate production were found to be much higher in HCC TRCs, compared to the corresponding cell lines, which is consistent with other studies [47]. However, the application of sulfarotene has not resulted in the decrease of glucose consumption and lactate production in HCC TRCs, suggesting that sulfarotene could not reverse sorafenib resistance through the glycolysis pathway (Figure S16). Overall, it would be interesting to reveal the plasticity of metabolism in liver CSCs and its contribution to sorafenib resistance. 


\section{Conclusions}

Taken together, our findings have identified sulfarotene as a potentially effective agent that selectively targets HCC tumor-repopulating cells, which in many aspects resemble cancer stem cells and contribute significantly to the recurrence and drug resistance of $\mathrm{HCC}$, and highlights SOS2 as a critical new oncogenic factor in association with RAR $\alpha$ and RAS that together form a novel signal nexus in HCC.

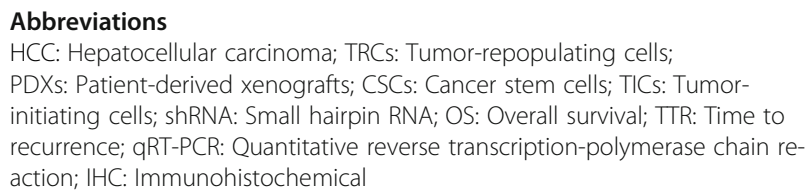

\section{Supplementary Information}

The online version contains supplementary material available at https://doi. org/10.1186/s13046-021-02085-4.

\section{Additional file 1: Supplementary Information.}

Additional file 2: Supplementary Data 1. The critical genes clustered in response to sulfarotene and in association with RARa.

Additional file 3: Supplementary Data 2. The association of SOS2 and associated neighboring genes.

\section{Acknowledgements}

We thank LC Biotech for aiding in bioinformatics analyses of RNA-Seq and ChIP-seq data.

\section{Authors' contributions}

$X C$, JLX and JWC carried out the conceptualization. FQ, WXQ and YZ carried out the biological experiments and sequencing. QLA participated in the chemical experiments. BWY, KQS, ZJY and QWR participated in the clinical experimental analyses and statistical evaluations. FQ and BN participated in statistical and bioinformatics analyses. FQ, XC and YDL carried out to draft the manuscript, and YDL, JLX, XC and JWC reviewed and edited the manuscript. The authors read and approved the final manuscript.

\section{Funding}

This study was funded by the Natural Science Foundation of Shanghai (No. 20ZR1410400 to X. Cao), National Natural Science Foundation of China (No. 81772590 and 81572395 to JL. Xia, No. 82173662 to X. Cao, No. 11902121 and 32071306 to JW. Chen). The Open Funding of Key Laboratory of Diagnosis and Treatment of Severe Hepato-pancreatic Diseases of Zhejiang Province (No. $2018 \mathrm{E} 10008$ to X. Cao), and CAS Interdisciplinary Innovation Team (No. JCTD-2019-07 to X. Cao).

\section{Availability of data and materials}

The obtained RNA-Seq and ChIP-Seq raw data were uploaded to the Sequence Read Archive (SRA) database of the National Center for Biotechnology Information (NCBI) (https://www.ncbi.nlm.nih.gov/), with an accession number of PRJNA673935. All data are available in the main text or in the supplementary materials.

\section{Declarations}

\section{Ethics approval and consent to participate}

The animal study protocols were performed in accordance with the Guide for the Care and Use of Laboratory Animals stipulated by the National Academy of Sciences and the National Institutes of Health (NIH publication 86 - 23, revised 1985) and approved by the Animal Care and Use Committee of Zhongshan Hospital, Fudan University, Shanghai, China. Each patient provided informed consent before they participated in the study. The study protocol was approved by the Ethics Committee of The First Affiliated Hospital of Wenzhou Medical University (Zhejiang, China, No. 123/2020).

\section{Consent for publication}

The authors declare that they agree to submit the article for publication.

\section{Competing interests}

All authors declare that no competing interests are associated with this study.

\section{Author details}

${ }^{1}$ Institute of Clinical Science, Zhongshan Hospital, Fudan University, 180 Fenglin Road, 200032 Shanghai, China. ${ }^{2}$ Liver Cancer Institute, Zhongshan Hospital, Fudan University, 180 Fenglin Road, 200032 Shanghai, China. ${ }^{3}$ Department of Oncology, Second Affiliated Hospital of Naval Medical University, 200003 Shanghai, China. ${ }^{4}$ Laboratory for Cellular Biomechanics and Regenerative Medicine, Department of Biomedical Engineering, College of Life Science and Technology, Huazhong University of Science and Technology, 1037 Luoyu Road, 430074 Wuhan, Hubei, China. ${ }^{5}$ The First Affiliated Hospital of Wenzhou Medical University, 325000 Wenzhou, Zhejiang, China. ${ }^{6}$ School of Pharmaceutical Sciences, Wenzhou Medical University, 325000 Wenzhou, Zhejiang, China. ${ }^{7}$ School of Life Sciences, Shanghai University, 200444 Shanghai, China.

Received: 20 July 2021 Accepted: 24 August 2021

Published online: 04 September 2021

\section{References}

1. Fitzmaurice C, Abate D, Abbasi N, Abbastabar H, Abd-Allah F, AbdelRahman O, et al. Global, Regional, and National Cancer Incidence, Mortality, Years of Life Lost, Years Lived With Disability, and Disability-Adjusted LifeYears for 29 Cancer Groups, 1990 to 2017: A Systematic Analysis for the Global Burden of Disease Study. JAMA Oncol. 2019:5(12):1749-68.

2. Gyawali B, Prasad V. Health policy: Me-too drugs with limited benefits - the tale of regorafenib for HCC. Nat Rev Clin Oncol. 2017;14(11):653-4.

3. Llovet JM, Montal R, Sia D, Finn RS. Molecular therapies and precision medicine for hepatocellular carcinoma. Nat Rev Clin Oncol. 2018;15(10):599616.

4. Magee JA, Piskounova E, Morrison SJ. Cancer stem cells: impact, heterogeneity, and uncertainty. Cancer Cell. 2012;21(3):283-96.

5. Ma S, Chan KW, Hu L, Lee TK, Wo JY, Ng IO, et al. Identification and characterization of tumorigenic liver cancer stem/progenitor cells. Gastroenterology. 2007;132(7):2542-56.

6. Liu J, Tan Y, Zhang H, Zhang Y, Xu P, Chen J, et al. Soft fibrin gels promote selection and growth of tumorigenic cells. Nat Mater. 2012;11(8):734-41.

7. Huang W, Hu H, Zhang Q, Wu X, Wei F, Yang F, et al. Regulatory networks in mechanotransduction reveal key genes in promoting cancer cell stemness and proliferation. Oncogene. 2019:38(42):6818-34.

8. Chen J, Cao X, An Q, Zhang Y, Li K, Yao W, et al. Inhibition of cancer stem cell like cells by a synthetic retinoid. Nat Commun. 2018:9(1):1406.

9. $\quad$ Tang $W, L v B$, Yang B, Chen Y, Yuan F, Ma L, et al. TREM2 acts as a tumor suppressor in hepatocellular carcinoma by targeting the PI3K/Akt/ $\beta$-catenin pathway. Oncogenesis. 2019;8(2):9.

10. Nazzal M, Sur S, Steele R, Khatun M, Patra T, Phillips N, et al. Establishment of a Patient-Derived Xenograft Tumor From Hepatitis C-Associated Liver Cancer and Evaluation of Imatinib Treatment Efficacy. Hepatology. 2020; 72(2):379-88.

11. Yang B, Li M, Tang W, Liu W, Zhang S, Chen L, et al. Dynamic network biomarker indicates pulmonary metastasis at the tipping point of hepatocellular carcinoma. Nat Commun. 2018;9(1):678.

12. Kanehisa M, Goto S. KEGG: kyoto encyclopedia of genes and genomes. Nucleic Acids Res. 2000;28(1):27-30.

13. Qin XY, Suzuki H, Honda M, Okada H, Kaneko S, Inoue I, et al. Prevention of hepatocellular carcinoma by targeting MYCN-positive liver cancer stem cells with acyclic retinoid. Proc Natl Acad Sci U S A. 2018;115(19):4969-74.

14. Tang W, Chen Z, Zhang W, Cheng Y, Zhang B, Wu F, et al. The mechanisms of sorafenib resistance in hepatocellular carcinoma: theoretical basis and therapeutic aspects. Signal Transduct Target Ther. 2020;5(1):87.

15. Wołoszynowska-Fraser MU, Kouchmeshky A, McCaffery P. Vitamin A and Retinoic Acid in Cognition and Cognitive Disease. Annu Rev Nutr. 2020:40: 247-72. 
16. Schwartz DM, Farley TK, Richoz N, Yao C, Shih HY, Petermann F, et al. Retinoic Acid Receptor Alpha Represses a Th9 Transcriptional and Epigenomic Program to Reduce Allergic Pathology. Immunity. 2019;50(1): $106-20 . e 10$.

17. Tan $Y$, Wang $X$, Song $H$, et al. A PML/RARa direct target atlas redefines transcriptional deregulation in acute promyelocytic leukemia. Blood. 2021; 137(11):1503-16.

18. Zhou G, Soufan O, Ewald J, Hancock REW, Basu N, Xia J. NetworkAnalyst 3.0: a visual analytics platform for comprehensive gene expression profiling and meta-analysis. Nucleic Acids Res. 2019;47(W1):W234-w41.

19. Otali D, Fredenburgh J, Oelschlager DK, Grizzle WE. A standard tissue as a control for histochemical and immunohistochemical staining. Biotech Histochem. 2016;91(5):309-26.

20. Sheffels E, Sealover NE, Wang C, Kim DH, Vazirani IA, Lee E, et al. Oncogenic RAS isoforms show a hierarchical requirement for the guanine nucleotide exchange factor SOS2 to mediate cell transformation. Sci Signal. 2018;11: 546.

21. Llovet JM, Kelley RK, Villanueva A, Singal AG, Pikarsky E, Roayaie S, et al. Hepatocellular carcinoma. Nat Rev Dis Primers. 2021;7(1):6.

22. Jiang MJ, Chen YY, Dai JJ, Gu DN, Mei Z, Liu FR, et al. Dying tumor cellderived exosomal miR-194-5p potentiates survival and repopulation of tumor repopulating cells upon radiotherapy in pancreatic cancer. $\mathrm{Mol}$ Cancer. 2020;19(1):68.

23. Masetti R, Vendemini F, Zama D, Biagi C, Gasperini P, Pession A. All-trans retinoic acid in the treatment of pediatric acute promyelocytic leukemia. Expert Rev Anticancer Ther. 2012;12(9):1191-204.

24. Yamashita T, Ji J, Budhu A, Forgues M, Yang W, Wang HY, et al. EpCAMpositive hepatocellular carcinoma cells are tumor-initiating cells with stem/ progenitor cell features. Gastroenterology. 2009;136(3):1012-24.

25. Matsushima-Nishiwaki R, Okuno M, Takano Y, Kojima S, Friedman SL, Moriwaki H. Molecular mechanism for growth suppression of human hepatocellular carcinoma cells by acyclic retinoid. Carcinogenesis. 2003; 24(8):1353-9.

26. Okita K, Izumi N, Ikeda K, Osaki Y, Numata K, Ikeda M, et al. Survey of survival among patients with hepatitis $C$ virus-related hepatocellular carcinoma treated with peretinoin, an acyclic retinoid, after the completion of a randomized, placebo-controlled trial. J Gastroenterol. 2015;50(6):667-74.

27. Pierre S, Bats AS, Chevallier A, Bui LC, Ambolet-Camoit A, Garlatti M, et al. Induction of the Ras activator Son of Sevenless 1 by environmental pollutants mediates their effects on cellular proliferation. Biochem Pharmacol. 2011;81(2):304-13.

28. Lu S, Jang H, Gu S, Zhang J, Nussinov R. Drugging Ras GTPase: a comprehensive mechanistic and signaling structural view. Chem Soc Rev. 2016;45(18):4929-52.

29. Frau M, Feo F, Pascale RM. Pleiotropic effects of methionine adenosyltransferases deregulation as determinants of liver cancer progression and prognosis. J Hepatol. 2013;59(4):830-41.

30. Singh AM, Reynolds D, Cliff T, Ohtsuka S, Mattheyses AL, Sun Y, et al. Signaling network crosstalk in human pluripotent cells: a Smad2/3regulated switch that controls the balance between self-renewal and differentiation. Cell Stem Cell. 2012;10(3):312-26.

31. Zhai X, Xue Q, Liu Q, Guo Y, Chen Z. Classifier of cross talk genes predicts the prognosis of hepatocellular carcinoma. Mol Med Rep. 2017;16(3):3253-61.

32. Hillig RC, Sautier B, Schroeder J, Moosmayer D, Hilpmann A, Stegmann CM, et al. Discovery of potent SOS1 inhibitors that block RAS activation via disruption of the RAS-SOS1 interaction. Proc Natl Acad Sci U S A. 2019; 116(7):2551-60.

33. Liceras-Boillos P, Jimeno D, García-Navas R, et al. Differential Role of the RasGEFs Sos 1 and Sos2 in Mouse Skin Homeostasis and Carcinogenesis. Mol Cell Biol. 2018;38(16):e00049-18.

34. Leung CON, Tong M, Chung KPS, Zhou L, Che N, Tang KH, et al. Overriding Adaptive Resistance to Sorafenib Through Combination Therapy With Src Homology 2 Domain-Containing Phosphatase 2 Blockade in Hepatocellular Carcinoma. Hepatology. 2020;72(1):155-68.

35. Zhao H, Cheng X, Yu J, Li Y. Stabilization of snail maintains the sorafenib resistance of hepatocellular carcinoma cells. Arch Biochem Biophys. 2021; 699:108754.

36. Chen BW, Zhou Y, Wei T, Wen L, Zhang YB, Shen SC, et al. IncRNA-POIR promotes epithelial-mesenchymal transition and suppresses sorafenib sensitivity simultaneously in hepatocellular carcinoma by sponging miR-1825p. J Cell Biochem. 2021;122(1):130-42.
37. Wang $H, X u H, M a F$, Zhan M, Yang $X$, Hua S, et al. Zinc finger protein 703 induces EMT and sorafenib resistance in hepatocellular carcinoma by transactivating CLDN4 expression. Cell Death Dis. 2020;11(4):225.

38. Chen W, Yang J, Zhang Y, Cai H, Chen X, Sun D. Regorafenib reverses HGFinduced sorafenib resistance by inhibiting epithelial-mesenchymal transition in hepatocellular carcinoma. FEBS Open Bio. 2019;9(2):335-47.

39. Xia S, Pan Y, Liang Y, Xu J, Cai X. The microenvironmental and metabolic aspects of sorafenib resistance in hepatocellular carcinoma. EBioMedicine. 2020;51:102610.

40. Huang Q, Li J, Xing J, Li W, Li H, Ke X, et al. CD147 promotes reprogramming of glucose metabolism and cell proliferation in HCC cells by inhibiting the p53-dependent signaling pathway. J Hepatol. 2014;61(4): 859-66.

41. Li S, Dai W, Mo W, Li J, Feng J, Wu L, et al. By inhibiting PFKFB3, aspirin overcomes sorafenib resistance in hepatocellular carcinoma. Int J Cancer. 2017; 141(12):2571-84.

42. Yoo JJ, Yu SJ, Na J, et al. Hexokinase-II Inhibition Synergistically Augments the Anti-tumor Efficacy of Sorafenib in Hepatocellular Carcinoma. Int J Mol Sci. 2019;20(6):1292.

43. Feng J, Wu L, Ji J, Chen K, Yu Q, Zhang J, et al. PKM2 is the target of proanthocyanidin B2 during the inhibition of hepatocellular carcinoma. J Exp Clin Cancer Res. 2019;38(1):204.

44. Zhang HL, Wang MD, Zhou X, Qin CJ, Fu GB, Tang L, et al. Blocking preferential glucose uptake sensitizes liver tumor-initiating cells to glucose restriction and sorafenib treatment. Cancer Lett. 2017;388:1-11.

45. Bort A, Sánchez BG, Mateos-Gómez PA, Vara-Ciruelos D, Rodríguez-Henche N, Díaz-Laviada I. Targeting AMP-activated kinase impacts hepatocellular cancer stem cells induced by long-term treatment with sorafenib. Mol Oncol. 2019;13(5):1311-31.

46. Wei Z, Jia J, Heng G, Xu H, Shan J, Wang G, et al. Sirtuin-1/Mitochondrial Ribosomal Protein S5 Axis Enhances the Metabolic Flexibility of Liver Cancer Stem Cells. Hepatology. 2019;70(4):1197-213.

47. Li Y, Luo S, Ma R, Liu J, Xu P, Zhang H, et al. Upregulation of cytosolic phosphoenolpyruvate carboxykinase is a critical metabolic event in melanoma cells that repopulate tumors. Cancer Res. 2015;75(7):1191-6.

\section{Publisher's Note}

Springer Nature remains neutral with regard to jurisdictional claims in published maps and institutional affiliations.

Ready to submit your research? Choose BMC and benefit from:

- fast, convenient online submission

- thorough peer review by experienced researchers in your field

- rapid publication on acceptance

- support for research data, including large and complex data types

- gold Open Access which fosters wider collaboration and increased citations

- maximum visibility for your research: over $100 \mathrm{M}$ website views per year

At BMC, research is always in progress.

Learn more biomedcentral.com/submissions 